\title{
Representation Formula for the Critical Points of the Tadjbakhsh-Odeh Functional and its Application
}

\author{
Kohtaro WATANABE* and Izumi TAKAGI ${ }^{\dagger}$ \\ * Department of Computer Science, National Defense Academy \\ 1-10-20 Hashirimizu, Yokosuka 239-8686, Japan \\ E-mail:wata@nda.ac.jp \\ † Mathematical Institute, Tohoku University \\ Aoba, Sendai 980-8578, Japan \\ E-mail: takagi@math.tohoku.ac.jp
}

Received May 23, 2007

Revised May 14, 2008

\begin{abstract}
In order to study the buckled states of an elastic ring under uniform pressure, Tadjbakhsh and Odeh [14] introduced an energy functional which is a linear combination of the total squared curvature (elastic energy) and the area enclosed by the ring. We prove that the minimizer of the functional is not a disk when the pressure is large, and its curvature can be expressed by Jacobian elliptic $\operatorname{cn}(\cdot)$ function. Moreover, the uniqueness of the minimizer is proven for certain range of the pressure.
\end{abstract}

Key words: Tadjbakhsh-Odeh functional, structure of the critical point, uniqueness of the minimizer, total squared curvature

\section{Introduction}

Let $\mathcal{S}$ denote the set of all smooth closed curves in the plane of length $L$ and of rotation number one. We fix an orthogonal coordinate system $(x, y)$ in the plane. For $\Gamma \in \mathcal{S}$, let $\Omega_{\Gamma}$ be the domain surrounded by $\Gamma$. Moreover, let $s$ be the arclength parameter and $\kappa(s)$ the curvature of $\Gamma$. As to the geometric inequalities involving the total squared curvature, Gage's inequality

$$
\frac{\pi L}{\operatorname{Area}\left(\Omega_{\Gamma}\right)} \geq \int_{\Gamma} \kappa^{2}(s) d s
$$

is known to hold if $\Gamma$ is a closed convex curve of class $C^{2}$ [8]. The equality holds if and only if $\Gamma$ is the circle of radius $L / 2 \pi$. Another example is the well-known classical variational problem called "elastica" [15]: Minimize

$$
\frac{1}{2} \int_{\Gamma} \kappa^{2}(s) d s,
$$

subject to the constraint that the length of $\Gamma$ is equal to $L$. If $\Gamma$ is restricted to the set $\mathcal{S}$, then by the Cauchy-Schwarz inequality, we immediately see that the minimizer of (2) is unique up to translation, and it is the circle of radius $L / 2 \pi$ again.

In this paper, we are interested in a geometric functional which involves the total squared curvature and the area, but has the non-disk type minimizer: 
Problem 1. Minimize

$$
E(\Gamma):=\frac{1}{2} \int_{\Gamma} \kappa^{2}(s) d s+p \operatorname{Area}\left(\Omega_{\Gamma}\right)
$$

over $\mathcal{S}$, where $\Gamma=\{(x(s), y(s)) \mid 0 \leq s \leq L\}, p$ is a positive number, and Area $\left(\Omega_{\Gamma}\right)$ is defined by

$$
\operatorname{Area}\left(\Omega_{\Gamma}\right):=\frac{1}{2} \int_{\Gamma}\left(x y_{s}-y x_{s}\right) d s
$$

We remark that the functional $E(\Gamma)$ was first considered by Tadjbakhsh and Odeh [14] to study the equilibrium states of an elastic inextensible ring (see Antman [2, pp. 101-110] for related studies of this subject). In particular, they showed the existence of the minimizer $\Gamma_{\gamma}$ (Lemma 1 below). The purpose of this paper is to derive an explicit formula for the critical points of the functional $E(\Gamma)$ and to prove the uniqueness of the minimizer when $0<p \leq 120 \pi^{3} / L^{3}$. The main result is stated as follows:

\section{Theorem 1.}

(i) If $p$ satisfies $0<p \leq 24 \pi^{3} / L^{3}$, then the functional $E(\Gamma)$ has a unique minimizer (up to translation), which is the circle of radius $L / 2 \pi$ (trivial solution).

(ii) If $24 \pi^{3} / L^{3}<p \leq 120 \pi^{3} / L^{3}$, then $E(\Gamma)$ has a unique minimizer $\Gamma_{\gamma}$ and it is not a circle. Indeed the curvature $\kappa(s)$ of $\Gamma_{\gamma}$ has the expression

$$
\kappa(s)=\frac{1}{a \operatorname{cn}(h s, m)+b}+c,
$$

where $\mathrm{cn}(\cdot, m)$ is the Jacobian elliptic cosine function, $h=8 K(m) / L$, $(K(m)$ is the complete elliptic integral of the first kind; see Definition 1) and for each $p$ in this range, $(a, b, c, m)$ is uniquely determined by the following set of equations:

$$
\left\{\begin{array}{l}
\text { (i) } a=-\sqrt{\frac{-1+4 b^{2} h^{2}(1-2 m)+\sqrt{\left(1-4 b^{2} h^{2}(1-2 m)\right)^{2}+64 b^{4} h^{4} m(1-m)}}{8 h^{2}(1-m)}}, \\
\text { (ii) } c=-\frac{1+\sqrt{\left(1-4 b^{2} h^{2}\right)^{2}+16 b^{2} h^{2} m}}{2 b}, \\
\text { (iii) } 2 b h^{4} \sqrt{\left(1-4 b^{2} h^{2}\right)^{2}+16 b^{2} h^{2} m}=p, \\
\text { (iv) } \int_{0}^{\frac{L}{4}} \kappa(s) d s=\frac{\pi}{2} .
\end{array}\right.
$$

As a matter of fact, we prove this theorem by obtaining the representation formula for all critical points of the functional $E(\Gamma)$; see Theorem 2. In particular, we obtain all the critical points for each value of $p$. By making use of the representation formula we might be able to investigate the local dynamics of the gradient 
flow of the Tadjbakhsh-Odeh functional in detail, and hence our results may be regarded as a first step to understand the global behavior of the gradient flow of this functional. See, e.g., [12] for recent development in this direction.

Fig. 10 illustrates the shapes of the minimizers for various values of $p$ (see (b), (c), (d), (e) of Fig. 10). On the other hand, Theorem 1 does not guarantee the minimality of case (f) of Fig. 10, because $120 \pi^{3} / L^{3}=15<17$ holds when $L=2 \pi$. Therefore, the problem of the uniqueness and the structure of the minimizer for $p>120 \pi^{3} / L^{3}$ still remains open.

We mention here another variational problem related to Problem 1: Minimize (2) subject to the constraints $\Gamma \in \mathcal{S}$ and $\operatorname{Area}\left(\Omega_{\Gamma}\right)=M$, where $M$ is a given positive constant. We call this problem the 2-D Canham problem; see Canham [5]. The minimizer of this problem describes the equilibrium states of a lipid membrane (see, e.g., Arreaga et al. [4], and Satake and Honda [13] for its dynamical aspects). In addition, the minimizer presents an example of domains which are determined by the eigenvalues of the Laplace operator, a positive result for Mark Kac's problem [10]; see [16]. Lately, Matsumoto, Murai and Yotsutani [11] studied the structures of critical points of this problem and announced that the critical point of mode $n$ is unique.

\section{Structure of critical points}

We begin by recalling some known facts on the existence of minimizers and critical points of the functional $E(\Gamma)$. Let $\theta(s)$ be the angle between a tangent $(d x(s) / d s, d y(s) / d s)$ at the point $(x(s), y(s)) \in \Gamma$ and the positive $x$-axis. Without loss of generality, we may assume that $(x(0), y(0))=0$ and $\theta(0)=0$. Then

$$
x(s)=\int_{0}^{s} \cos \theta(t) d t \text { and } y(s)=\int_{0}^{s} \sin \theta(t) d t .
$$

Moreover $\kappa(s)=\theta^{\prime}(s)$ and $x(s) y^{\prime}(s)-x^{\prime}(s) y(s)=\int_{0}^{s} \sin (\theta(s)-\theta(t)) d t$. Hence, $E(\Gamma)$ can be expressed in terms of $\theta$ and $\theta^{\prime}$ only. The following existence result was obtained by Tadjbakhsh and Odeh [14].

Lemma 1 ([14, Proposition 2]). Let $F$ be the set of all functions $\theta \in H^{1}(0, L)$ satisfying

$$
\begin{aligned}
& x(0)=x(L)=0, \quad y(0)=y(L)=0, \\
& \theta(0)=0, \quad \theta(L)=2 \pi .
\end{aligned}
$$

Then the functional

$$
E(\theta):=\frac{1}{2} \int_{0}^{L}\left(\theta^{\prime}\right)^{2}(s) d s+\frac{p}{2} \int_{0}^{L} \int_{0}^{s} \sin (\theta(s)-\theta(\xi)) d \xi d s
$$

attains its minimum in $F$. 
Thus Problem 1 has at least one minimizer in some subset $F$ of $H^{1}(0, L)$, where $H^{1}(0, L)$ is the usual Sobolev space of first order. As for the smoothness of the minimizer, we have the following result.

Lemma 2 ([16, Lemma 3.4]). Let $\theta_{0} \in F$ be a minimizer of the functional $E$. Then $\theta_{0}$ is a smooth periodic function with period $T=L / n$. More precisely,

(i) $\theta_{0} \in C^{\infty}\left[0, \frac{L}{n}\right]$,

(ii) $\theta_{0+}^{(m)}(0)=\theta_{0-}^{(m)}\left(\frac{L}{n}\right)$ for all $m \in \mathbb{N}$,

where $\theta_{0+}^{(m)}$ and $\theta_{0-}^{(m)}$ denote the right and left derivatives of order $m$, respectively. Moreover, $\kappa=\partial_{s} \theta_{0}$ satisfies the following Euler-Lagrange equation:

$$
\kappa_{s s}+\frac{1}{2} \kappa^{3}-q_{1} \kappa-p=0
$$

which has the first integral

$$
\frac{1}{2}\left(\kappa_{s}\right)^{2}+\frac{1}{8} \kappa^{4}-\frac{1}{2} q_{1} \kappa^{2}-p \kappa=q_{2} .
$$

Here, $q_{1}$ and $q_{2}$ are the constants to be chosen so that the following conditions are satisfied:

$$
\begin{gathered}
\kappa_{s}(0)=\kappa_{s}\left(\frac{L}{2 n}\right)=0 \\
\int_{0}^{\frac{L}{2 n}} \kappa(s) d s=\frac{\pi}{n}
\end{gathered}
$$

for some positive integer $n$.

Proof. See, Tadjbakhsh and Odeh [14, p. 61, p. 64] or Watanabe [16, pp. 453-457] (In [14, p. 61], conditions (13) and (14) are shown without proof. We remark that the smoothness and periodicity of $\theta_{0}$ are necessary for the derivation of (13) and (14)).

By the general property of the equation (12) (see Arnold [3, Section 12]), any solution $\kappa$ is symmetric about the half period $T / 2=L /(2 n)$. Hence possible shapes of the minimizers are like Fig. 1. We obtain also from (12) that for any $p>0$, equations (12) to (14) always have the trivial solution $\kappa=2 \pi / L$, by suitably choosing $q_{1}$ and $q_{2}$. For $p>24 \pi^{3} / L^{3}$, the trivial solution is unstable:

Lemma 3 ([14, Theorem 2]). For $p>24 \pi^{3} / L^{3}$, the trivial solution $\kappa=2 \pi / L$ is always unstable, i.e., the circle $x^{2}+(y-2 \pi / L)^{2}=(2 \pi / L)^{2}$ is not a minimizer. 


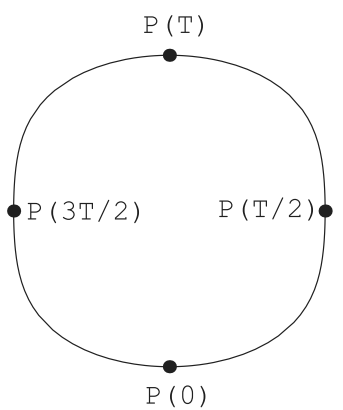

(a)

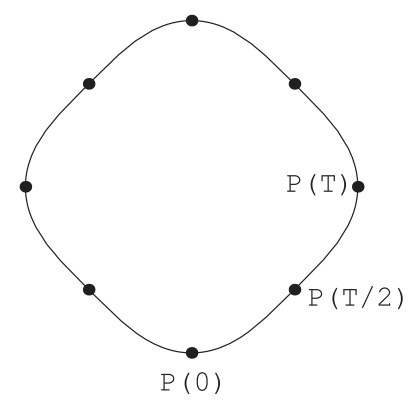

(a)

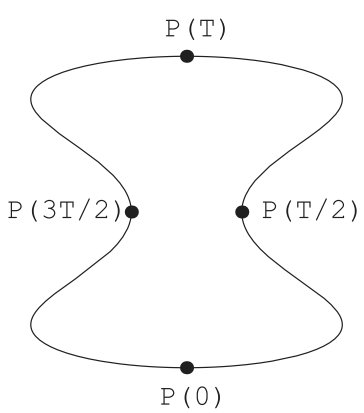

(b)

$\mathrm{T}=\mathrm{L} / 2$

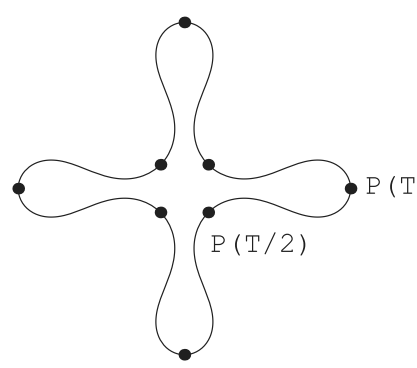

$P(0)$

(b)

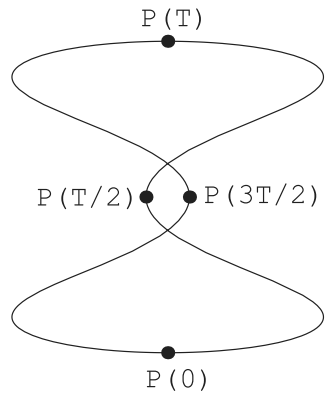

(c)

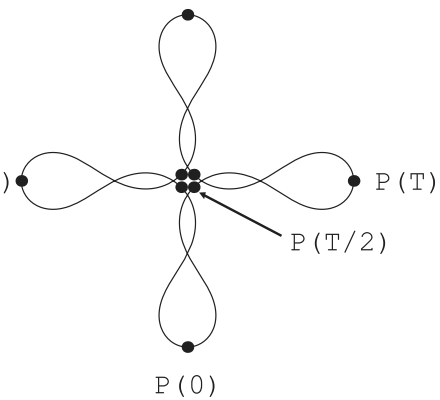

(c)

$\mathrm{T}=\mathrm{L} / 4$

Fig. 1. Possible shapes of minimizers for $T=L / 2$ and $T=L / 4$. (a) $\Omega_{\Gamma_{\gamma}}$ is convex $(k(0)>0$ and $k(T / 2) \geq 0)$, (b) $\Omega_{\Gamma_{\gamma}}$ is non-convex $(k(0)>0$ and $k(T / 2)<0)$, (c) $\Omega_{\Gamma_{\gamma}}$ is not simple.

The following lemma is deduced from the smoothness of minimizers:

Lemma 4. Let $\theta_{0}$ be a minimizer of the functional $E$. Then the period $T$ of $\theta_{0}$ must be either $L /(2 k)(k \in \mathbb{N})$ or $L$. Hence the region, $\Omega_{\Gamma_{0}}$ generated by $\theta_{0}$ must have an even number (or only one) of the axes of symmetry.

Proof. Suppose that $\theta_{0}$ has the period $T=L /(2 k+1), k \in \mathbb{N}$, and let us define $\tilde{\theta}_{0}$ by

$$
\tilde{\theta}_{0}(s):= \begin{cases}\theta_{0}(s), & \text { if } 0 \leq s \leq \frac{L}{2}, \\ \pi+\theta_{0}\left(s-\frac{L}{2}\right), & \text { if } \frac{L}{2} \leq s \leq L .\end{cases}
$$


Since $\tilde{\theta}_{0}$ is continuous at $s=L / 2$ and $s=0$, it is easy to see that $\tilde{\theta}_{0} \in F$. Moreover, $E\left(\tilde{\theta}_{0}\right)=E\left(\theta_{0}\right)$, because

$$
\int_{\frac{L}{2}}^{L}\left(\partial_{s} \tilde{\theta}_{0}\right)^{2}(s) d s=\int_{\frac{L}{2}}^{L}\left(\partial_{s} \theta_{0}\right)^{2}(s) d s,
$$

and

$$
\begin{aligned}
\int_{\frac{L}{2}}^{L} \int_{0}^{s} \sin \left(\tilde{\theta}_{0}(s)-\tilde{\theta}_{0}(\xi)\right) d \xi d s= & \int_{\frac{L}{2}}^{L} \int_{0}^{\frac{L}{2}} \sin \left(\pi+\theta_{0}\left(s-\frac{L}{2}\right)-\theta_{0}(\xi)\right) d \xi d s \\
& +\int_{\frac{L}{2}}^{L} \int_{\frac{L}{2}}^{s} \sin \left(\theta_{0}\left(s-\frac{L}{2}\right)-\theta_{0}\left(\xi-\frac{L}{2}\right)\right) d \xi d s \\
= & \int_{0}^{\frac{L}{2}} \int_{0}^{u} \sin \left(\theta_{0}(u)-\theta_{0}(v)\right) d v d u \\
= & \int_{\frac{L}{2}}^{L} \int_{0}^{s} \sin \left(\theta_{0}(s)-\theta_{0}(\xi)\right) d \xi d s
\end{aligned}
$$

Observe that the last equality results from

$$
\begin{aligned}
& \int_{\frac{L}{2}}^{L} \int_{0}^{s} \sin \left(\theta_{0}(s)-\theta_{0}(\xi)\right) d \xi d s \\
& =\int_{\frac{L}{2}}^{L} \int_{0}^{s} \sin \left(-\theta_{0}(L-s)+\theta_{0}(L-\xi)\right) d \xi d s \quad\left(\theta_{0}(L-s)=2 \pi-\theta_{0}(s)\right) \\
& =\int_{0}^{\frac{L}{2}} \int_{0}^{u} \sin \left(\theta_{0}(u)-\theta_{0}(v)\right) d v d u
\end{aligned}
$$

Therefore, $\tilde{\theta}_{0}$ is also a minimizer of $E$. This contradicts the smoothness of the minimizer (Lemma 2), since $\partial_{s} \tilde{\theta}_{0}(s)$ is not continuous at $s=L / 2$.

Here, we recall the definition of the Jacobian elliptic functions.

Definition 1 ([1, p. 569]). Let $m$ be a real number satisfying $0 \leq m<1$. The Jacobian elliptic function $\operatorname{sn}(u, m)$ of modulus $\sqrt{m}$ is defined by the inverse function of the indefinite integral

$$
s=\int_{0}^{z} \frac{1}{\sqrt{\left(1-x^{2}\right)\left(1-m x^{2}\right)}} d x, \quad(-1 \leq z \leq 1) .
$$

The complete elliptic integral of the first kind $K(m)$ is defined as

$$
K(m):=\int_{0}^{1} \frac{1}{\sqrt{\left(1-x^{2}\right)\left(1-m x^{2}\right)}} d x .
$$

Hence, $\operatorname{sn}(\cdot, m)$ is defined on the interval $[-K(m), K(m)]$. Associate functions $\operatorname{cn}(\cdot, m)$ and $\operatorname{dn}(\cdot, m)$ are defined as $\operatorname{cn}(\cdot, m)=\sqrt{1-\operatorname{sn}^{2}(\cdot, m)}$ and $\operatorname{dn}(\cdot, m)=\sqrt{1-m \mathrm{sn}^{2}(\cdot, m)}$ respectively on the interval $[-K(m), K(m)]$. The domain of definition of these elliptic functions is extended to $[0,4 K(m)]$ by using 
the relation $\operatorname{sn}(z+2 K(m), m)=-\operatorname{sn}(z, m), \operatorname{cn}(z+2 K(m), m)=-\operatorname{cn}(z, m)$, and $\operatorname{dn}(z+2 K(m), m)=\operatorname{dn}(z, m)$. For the sake of simplicity, we write the Jacobian elliptic functions $\operatorname{sn}(\cdot, m), \operatorname{cn}(\cdot, m), \operatorname{dn}(\cdot, m)$ as $\operatorname{sn}(\cdot), \operatorname{cn}(\cdot), \operatorname{dn}(\cdot)$ respectively if there is no fear of confusion.

LEMMA 5. Any solution of (12) must be one of the following three forms:

$$
\kappa(s)= \begin{cases}\text { (i) } & \frac{1}{a \operatorname{cn}(h s)+b}+c, \\ \text { (ii) } & \frac{1}{a \operatorname{sn}(K(m)-h s)+b}+c, \\ \text { (iii) } & a \operatorname{cn}(h s),\end{cases}
$$

where $a, b, c, m(0 \leq m<1)$ and $h$ are certain real constants.

Proof. Since it is routine but rather lengthy, we shall give the proof in Appendix A.

We observe that the function $\operatorname{sn}(K(m)-h s)$ appearing in Case (ii) can be expressed in terms of the elliptic dn function.

LEMma 6. For arbitrary positive numbers $h$ and $m$ with $0<m<1$,

$$
\operatorname{sn}(K(m)-h s, m)=\frac{\left(1+m^{-\frac{1}{4}}\right) \operatorname{dn}\left(\frac{h\left(1+m^{\frac{1}{4}}\right)^{2} s}{2}, \mu(m)\right)+1-m^{-\frac{1}{4}}}{\left(1+m^{\frac{1}{4}}\right) \operatorname{dn}\left(\frac{h\left(1+m^{\frac{1}{4}}\right)^{2} s}{2}, \mu(m)\right)+1-m^{\frac{1}{4}}},
$$

where

$$
\mu(m):=\frac{8 m^{\frac{1}{4}}\left(1+m^{\frac{1}{2}}\right)}{\left(1+m^{\frac{1}{4}}\right)^{4}}
$$

holds.

Proof. See Appendix B.

From Lemmas 5 and 6 , it turns out that there are essentially two possibilities to be considered, as stated in the following lemma.

Lemma 7. Any nontrivial solutions of (12) to (14) must be one of the following two forms:

$$
\left\{\begin{array}{l}
\text { (i) } \frac{1}{a \operatorname{cn}\left(\frac{4 n K(m) s}{L}\right)+b}+c, \\
\text { (ii) } \frac{1}{a \operatorname{dn}\left(\frac{2 n K(m) s}{L}\right)+b}+c, \quad(m \neq 0),
\end{array}\right.
$$

where $a, b, c$, and $m(0 \leq m<1)$ are certain real constants. 
Proof. If a function of the form (i)-(iii) in (16) satisfies (13), then $h$ equals $4 n K(m) / L$ for some $n \in \mathbb{N}$. This shows that (19) (i) is a possible form of a solution. Next, we assume that $\kappa(s)$ is of the form (ii) in (16). Because ${ }^{1}$

$$
K(m)\left(1+m^{\frac{1}{4}}\right)^{2}=K\left(8 m^{\frac{1}{4}}\left(1+m^{\frac{1}{2}}\right) /\left(1+m^{\frac{1}{4}}\right)^{4}\right),
$$

we have

$$
\operatorname{dn}\left(\frac{2 n K(m)\left(1+m^{\frac{1}{4}}\right)^{2} s}{L}, \mu(m)\right)=\operatorname{dn}\left(\frac{2 n K(\mu(m)) s}{L}, \mu(m)\right) .
$$

In addition, $\mu(m)=8 m^{\frac{1}{4}}\left(1+m^{\frac{1}{2}}\right) /\left(1+m^{\frac{1}{4}}\right)^{4}$ is a bijection from $[0,1]$ to $[0,1]$. Thus, when $m \neq 0$, Case (ii) of (16) is equivalent to Case (ii) of (19). If $m=0$, then

$$
\operatorname{sn}(K(0)-h(0) s, 0)=\operatorname{sn}\left(\frac{\pi}{2}-\frac{2 n \pi s}{L}, 0\right)=-\cos \left(\frac{2 n \pi s}{L}\right) .
$$

Therefore, this case is included in (19) (i). Finally, we show that Case (iii) of (16) is ruled out. Indeed,

$$
\int_{0}^{\frac{L}{2 n}} \operatorname{cn}\left(\frac{4 n K(m) s}{L}\right) d s=0
$$

implies that (14) is never satisfied. This completes the proof.

REMARK 1. We prefer the expression Case (ii) of Lemma 7 to Case (ii) of Lemma 5 , since it simplifies the study of the behavior of the quantity

$$
\int_{0}^{\frac{L}{2 n}} \kappa(s) d s
$$

as $m$ approaches 1 (see Lemma 29 bellow).

The next theorem clarifies the structure of the critical points of $E(\Gamma)$ :

\section{Theorem 2.}

(i) If $n=1$, then for any $p>0$, there exists no nontrivial solution of (12) to $(14)$.

(ii) If $n \geq 2$ and $0<p \leq 8 \pi^{3}\left(n^{2}-1\right) / L^{3}$, then (12) to (14) have no nontrivial solution with period $L / n$.

\footnotetext{
${ }^{1}$ We acknowledge that Professor Yoshihiro Ônishi of Iwate University kindly suggested us that identity (20) can be deduced easily from a formula in collected work of C.G.J. Jacobi [9, p. 521].
} 
(iii) If $n \geq 2$ and $8 \pi^{3}\left(n^{2}-1\right) / L^{3}<p$, then (12) to (14) have a unique nontrivial solution. Moreover, the structure of the nontrivial solution is as follows:

$\kappa(s)$
$= \begin{cases}\frac{1}{a_{1} \operatorname{cn}\left(\frac{4 n K(m) s}{L}\right)+b_{1}}+c_{1} & \text { if } \frac{8 \pi^{3}}{L^{3}}\left(n^{2}-1\right) \leq p<\frac{8 \pi^{3}}{L^{3}}(n-1)(2 n-1)(3 n-1), \\ \frac{1}{a_{2} \operatorname{dn}\left(\frac{2 n K(m) s}{L}\right)+b_{2}}+c_{2} & \text { if } \frac{8 \pi^{3}}{L^{3}}(n-1)(2 n-1)(3 n-1)<p,\end{cases}$

where $\left(a_{1}, b_{1}, c_{1}, m\right)$ is the unique solution of the equations (6) (i), (6) (ii), (6) (iii) and

$$
\int_{0}^{\frac{L}{2 n}} \kappa(s) d s=\frac{\pi}{n},
$$

while $\left(a_{2}, b_{2}, c_{2}, m\right)$ is the unique solution of the following equations:

$$
\left\{\begin{array}{l}
\text { (i) } a_{2}=-\sqrt{\frac{1+4\left(b_{2}\right)^{2} h^{2}(2-m)-\sqrt{1+8\left(b_{2}\right)^{2} h^{2}(2-m)+16\left(b_{2}\right)^{4} h^{4} m^{2}}}{8 h^{2}(1-m)}} \\
\text { (ii) } c_{2}=-\frac{1+\sqrt{1+8\left(b_{2}\right)^{2} h^{2}(2-m)+16\left(b_{2}\right)^{4} h^{4} m^{2}}}{2 b_{2}} \\
\text { (iii) } 2 b_{2} h^{4} m^{2} \sqrt{1+8\left(b_{2}\right)^{2} h^{2}(2-m)+16\left(b_{2}\right)^{4} h^{4} m^{2}}=p
\end{array}\right.
$$

together with (23). Here $h=2 n K(m) / L$.

As an immediate corollary to Theorem 2, we know the exact number of critical points for each value of $p>0$ :

Corollary 1. If

$$
\frac{8 \pi^{3}}{L^{3}}\left(n^{2}-1\right) \leq p<\frac{8 \pi^{3}}{L^{3}} n(n+2)
$$

for some positive integer $n$, then the functional $E(\Gamma)$ has exactly one trivial critical point (i.e., the circle) and $n-1$ nontrivial critical points, up to rotation and translation.

In Section 4, we investigate in detail the question of existence and uniqueness of solutions to the system of transcendental equations (6) (i), (ii), (iii) and (23) (also (24) (i), (ii), (iii) and (23)). 


\section{Proof of Theorem 1}

Assuming Theorem 2, we prove Theorem 1.

Proof. When $0<p \leq 24 \pi^{3} / L^{3}$, by Theorem 2 (i) and (ii), there is no nontrivial solution for all $n \in \mathbb{N}$. On the other hand, for all $p>0$, we always have a trivial solution $\kappa \equiv 2 \pi / L$ where $q_{1}$ and $q_{2}$ are uniquely determined by (11) and (12). Moreover, by Lemma 1 , this trivial solution is the minimizer for $0<p \leq 24 \pi^{3} / L^{3}$. When $24 \pi^{3} / L^{3}<p \leq 120 \pi^{3} / L^{3}$, the trivial solution is unstable by Lemma 3 . Hence it is not a minimizer. On the other hand, by Theorem 2 , for $p$ in this range, nontrivial solutions exist only for (modes) $n=2$ or 3 ; see Fig. 2. However, by Lemma 4 , nontrivial solutions of mode 3 cannot be a minimizer. Therefore the nontrivial solution of mode 2 is the minimizer, and it has the expression of the form (5).

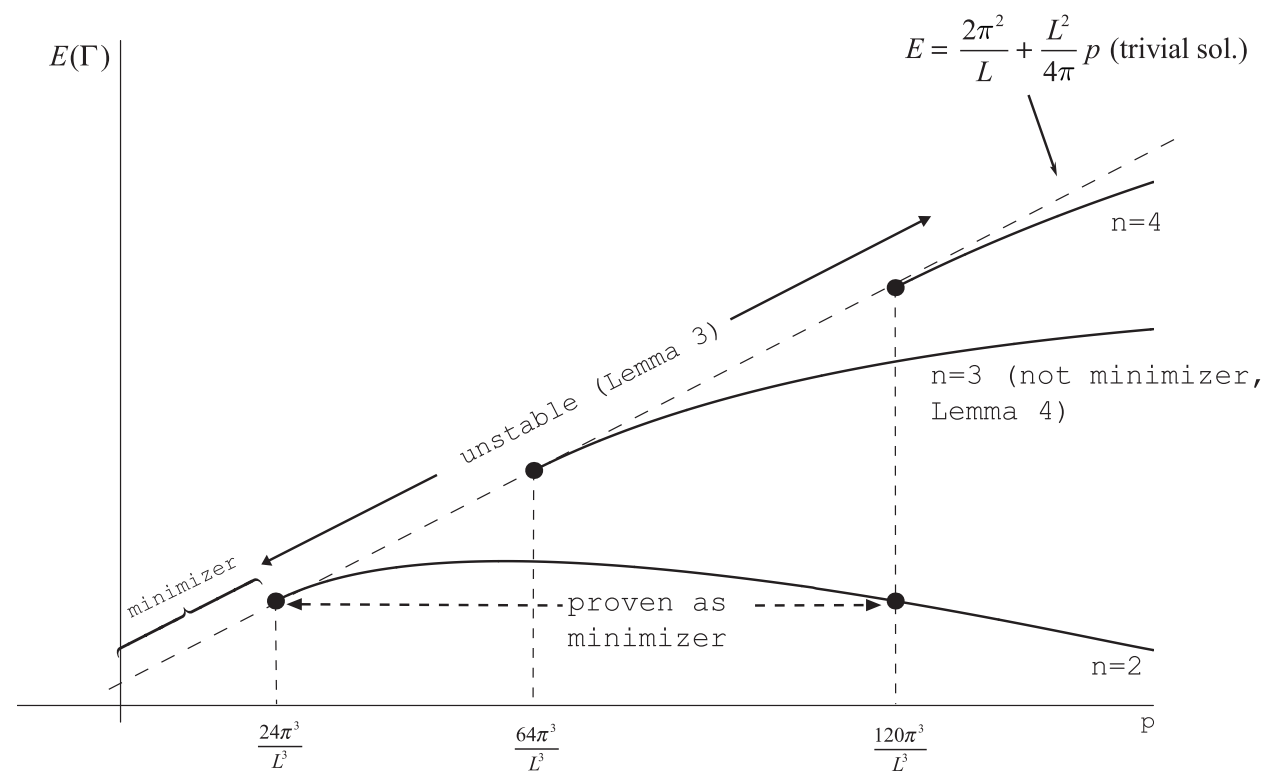

Fig. 2. Energy curves of stationary solutions. Bold lines are the energy of nontrivial solutions which have periods $T=L / 2, L / 3, L / 4$ (modes $n=2,3,4$ ) respectively. The dotted line represents the energy of the trivial solution.

It thus remains to prove Theorem 2 .

\section{Proof of Theorem 2}

\subsection{Case of (19) (i)}

First we consider the case where $\kappa(s)$ is of the form (19) (i).

LEMMA 8. Let

$$
\kappa(s)=\frac{1}{a \operatorname{cn}(h s)+b}+c \quad \text { with } h=\frac{4 n K(m)}{L}
$$


satisfy (12) and (13). Then $a, b$, and $c$ satisfy either

$$
\left\{\begin{array}{l}
\text { (i) } a= \pm \sqrt{\frac{-1+4 b^{2} h^{2}(1-2 m)+\sqrt{\left(1-4 b^{2} h^{2}(1-2 m)\right)^{2}+64 b^{4} h^{4} m(1-m)}}{8 h^{2}(1-m)}} \\
\text { (ii) } c=-\frac{1+\sqrt{\left(1-4 b^{2} h^{2}\right)^{2}+16 b^{2} h^{2} m}}{2 b}, \\
\text { (iii) } 2 b h^{4} \sqrt{\left(1-4 b^{2} h^{2}\right)^{2}+16 b^{2} h^{2} m}=p,
\end{array}\right.
$$

or

$$
\left\{\begin{array}{l}
\text { (i) } a= \pm \sqrt{\frac{-1+4 b^{2} h^{2}(1-2 m)-\sqrt{\left(1-4 b^{2} h^{2}(1-2 m)\right)^{2}+64 b^{4} h^{4} m(1-m)}}{8 h^{2}(1-m)}}, \\
\text { (ii) } c=\frac{-1+\sqrt{\left(1-4 b^{2} h^{2}\right)^{2}+16 b^{2} h^{2} m}}{2 b}, \\
\text { (iii) }-2 b h^{4} \sqrt{\left(1-4 b^{2} h^{2}\right)^{2}+16 b^{2} h^{2} m}=p .
\end{array}\right.
$$

Proof. Recall that $\kappa(s)$ satisfies the Euler-Lagrange equation (11). Substituting (19) (i) to (11), and using the relations

$$
\begin{aligned}
& \operatorname{sn}^{2}(h s)=1-\operatorname{cn}^{2}(h s), \\
& \operatorname{dn}^{2}(h s)=(1-m)+m \operatorname{cn}^{2}(h s),
\end{aligned}
$$

we obtain the following system of algebraic equations by putting the coefficients of $\mathrm{cn}^{k}(h s)(k=0,1,2,3)$ zero:

$$
\begin{aligned}
& 3 a c+6 a b c^{2}+3 a b^{2} c^{3}+2 a b h^{2}-4 a b h^{2} m-6 a b^{2} p-4 a b q_{1}-6 a b^{2} c q_{1}=0, \\
& 3 a^{2} c^{2}+3 a^{2} b c^{3}-2 a^{2} h^{2}+4 a^{2} h^{2} m-6 a^{2} b p-2 a^{2} q_{1}-6 a^{2} b c q_{1}=0 \\
& a^{3} c^{3}+4 a b h^{2} m-2 a^{3} p-2 a^{3} c q_{1}=0 \\
& 1+3 b c+3 b^{2} c^{2}+b^{3} c^{3}+4 a^{2} h^{2}-4 a^{2} h^{2} m-2 b^{3} p-2 b^{2} q_{1}-2 b^{3} c q_{1}=0 .
\end{aligned}
$$

We here remark that this system of equations was first considered by G.F. Carrier [6] more than half a century ago, but further analysis, as we see in the following, does not seem to have been carried out. Since we know $b \neq 0$ from the expressions for $\kappa(s)$ (see (110), (114), (116), (B-1), (B-2), and (B-3) of Appendix A), solving (27) and $(28)$ with respect to $\left(p, q_{1}\right)$, we have

$$
\begin{aligned}
p & =-\frac{1}{2 b}\left(c+3 b c^{2}+2 b^{2} c^{3}+2 b h^{2}+4 b^{2} c h^{2}-4 b h^{2} m-8 b^{2} c h^{2} m\right), \\
q_{1} & =\frac{-\left(-3 c-3 b c^{2}-4 b h^{2}+8 b h^{2} m\right)}{2 b} .
\end{aligned}
$$


Substituting these into (29) and (30), we have

$$
\begin{aligned}
& \frac{a\left(a^{2} c+2 a^{2} b h^{2}-4 a^{2} b h^{2} m+4 b^{3} h^{2} m\right)}{b^{2}}=0, \\
& 1+b c-4 a^{2} h^{2}(-1+m)+2 b^{2} h^{2}(-1+2 m)=0 .
\end{aligned}
$$

A solution (a,c) with $a \neq 0$ of (33) and (34) satisfies either (i), (ii) of (25) or (i), (ii) of (26). Substituting it into (31) leads to (25) (iii) or (26) (iii).

Clearly, the following lemma holds.

Lemma 9. For $0<m<1$, the right-hand side of (26) (i) is not a real number.

Therefore, we have only to consider (25) (i), (25) (ii), (25) (iii). Moreover, without loss of generality, we may assume $a \leq 0$ because of $\operatorname{cn}(z+2 K(m))=$ $-\operatorname{cn}(z)$. It is easy to check that $b>0$ and $b+a>0$, hence $\kappa(s)$ is defined for all $s \in[0, L]$. Let us put $u:=b^{2} h^{2}$. Then by (25) (iii), we see that $u$ may be a triple valued function of $m$. Hence, $a=a(m), b=b(m)$ and $c=c(m)$ are considered to be multi-valued functions of $m$.

Lemma 10. If $a(m)=0$, then $m=0$.

Proof. First, we observe that $b \neq 0$ (by Appendix A), and moreover $m \neq 1$. For, if $m=1$, then $h(1)=4 n K(1) / L=\infty$, so $\kappa(s)$ never satisfies (12)-(14) of Lemma 2. Now, let $a=0$. Then by (25) (i), we have $64 b^{4} h^{2} m(1-m)=0$ and hence, $m=0$.

Definition 2. Let $\kappa(s ; m)$ be given by

$$
\kappa(s ; m)=\frac{1}{a(m) \operatorname{cn}\left(\frac{4 n K(m) s}{L}\right)+b(m)}+c(m) .
$$

Using (35), we define a multi-valued function $F$ of $m$ by

$$
F(m):=\int_{0}^{\frac{L}{2 n}} \kappa(s ; m) d s .
$$

Our next job is to find positive $m^{*}$ satisfying (14), i.e., $F\left(m^{*}\right)=\pi / n$. If such $m^{*}$ exists, we have a nontrivial solution by Lemma 10. Here, let us take a look at numerical examples to see how the function $F$ behaves for various values of $p$. Fig. 3 shows the behavior of $F$ for various $p$ when $n=2$ and $L=2 \pi$. From Fig. 3, we guess that equation $F(m)=\pi / 2$

(i) does not have any solution, if $0<p<3$ (Fig. 3 (a)),

(ii) has a unique solution, if $3 \leq p \leq 15$ (Fig. 3 (b)-(g)),

(iii) again does not have any solution, if $15<p$ (Fig. $3(\mathrm{~h})$ ).

Note that we have in this case $24 \pi^{3} / L^{3}=3$ and $120 \pi^{3} / L^{3}=15$. In the following we prove that $y=F(m)$ behaves like Fig. 3 for arbitrary $n \in \mathbb{N}$. We begin by expressing $F$ in terms of the complete elliptic integrals of the first and third kind. 


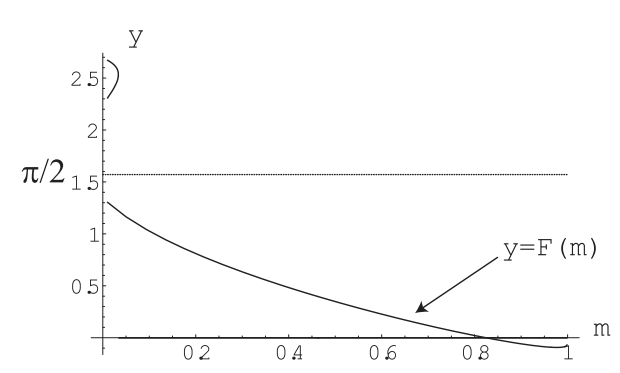

(a) $\mathrm{p}=2.8$

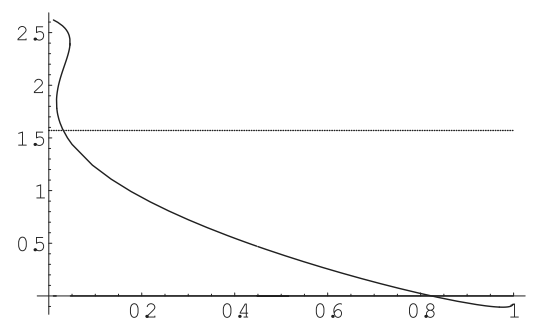

(c) $\mathrm{p}=3.2$

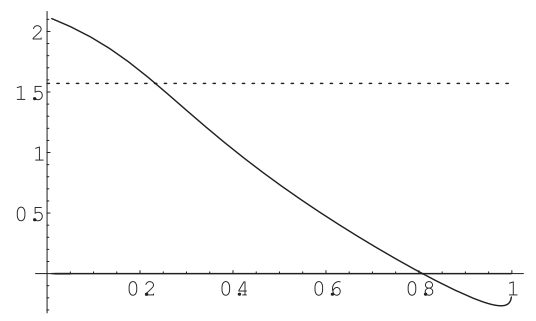

(e) $\mathrm{p}=8$

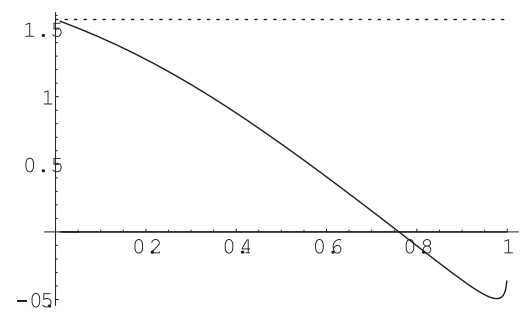

(g) $\mathrm{p}=15$

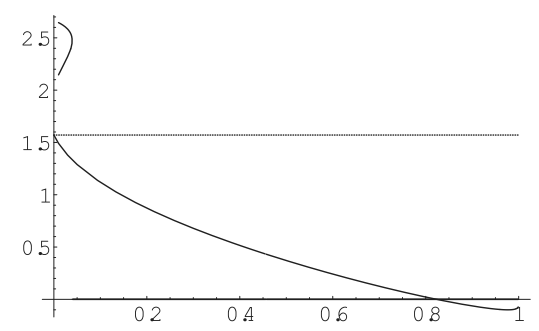

(b) $p=3$

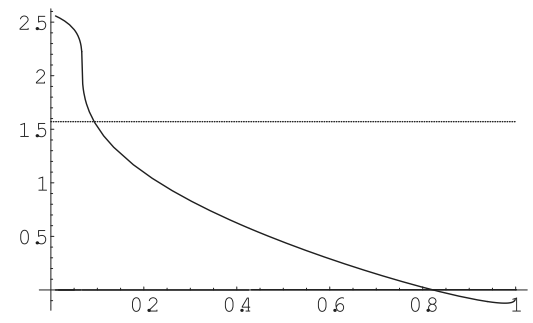

(d) $p=3.7$

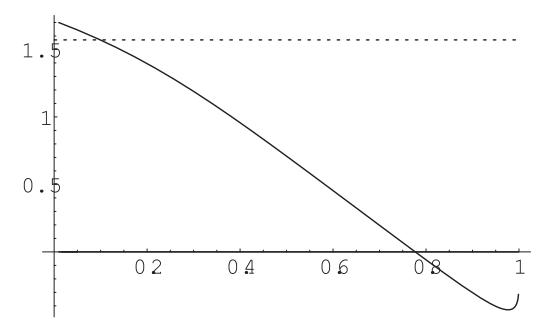

(f) $\mathrm{p}=13$

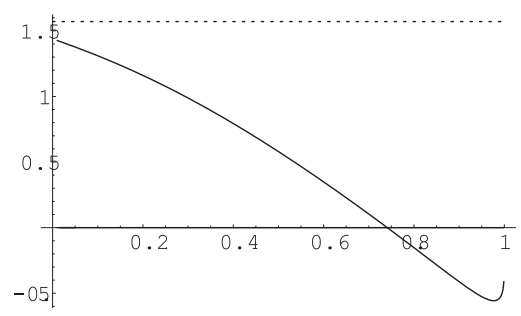

(h) $p=17$

Fig. 3. The behavior of $y=F(m)$ for various $p$ when $n=2$ and $L=2 \pi$. 
Lemma 11. Let $F(m)$ be given by (36). Then, we have

$$
F(m)=\frac{L b}{2 n\left(b^{2}-a^{2}\right)}\left(\frac{\Pi\left(\frac{a^{2}}{b^{2}-a^{2}}, m\right)}{K(m)}+\frac{\left(b^{2}-a^{2}\right) c}{b}\right),
$$

where $\Pi(\cdot, \cdot)$ is the complete elliptic integral of the third kind $([1, p .590])$ defined by

$$
\Pi(l, m):=\int_{0}^{1} \frac{1}{\left(1-l x^{2}\right) \sqrt{\left(1-x^{2}\right)\left(1-m x^{2}\right)}} d x .
$$

Proof. By a straightforward computation we obtain

$$
\begin{aligned}
& \int_{0}^{\frac{L}{2 n}} \frac{1}{a \operatorname{cn}(h s)+b} d s \\
& =\frac{1}{h} \int_{0}^{2 K(m)} \frac{1}{a \operatorname{cn}(t)+b} d t \\
& =-\frac{1}{h} \int_{-1}^{1} \frac{1}{(a x+b) \sqrt{\left(1-x^{2}\right)\left(1-m+m x^{2}\right)}} d x \quad(\text { putting } x=\operatorname{cn}(t)) \\
& =\frac{1}{h} \int_{0}^{1}\left(\frac{1}{a x+b}+\frac{1}{-a x+b}\right) \frac{1}{\sqrt{\left(1-x^{2}\right)\left(1-m+m x^{2}\right)}} d x \\
& =\frac{1}{h} \int_{0}^{1} \frac{1}{b^{2}-a^{2}+a^{2} z^{2}} \frac{1}{\sqrt{\left(1-z^{2}\right)\left(1-m z^{2}\right)}} d z \quad\left(\text { with } x^{2}=1-z^{2}\right) \\
& =\frac{L b}{2 n\left(b^{2}-a^{2}\right)} \frac{\Pi\left(-\frac{a^{2}}{b^{2}-a^{2}}, m\right)}{K(m)} .
\end{aligned}
$$

We can estimate how fast $b(m)$ vanishes as $m \rightarrow 1$ by the following lemma.

Lemma 12. Suppose $1 / 2 \leq m<1$. Then, the following estimate holds:

$$
b(m) \leq \frac{p}{2 h^{4}(m)} .
$$

In particular, we obtain

$$
\lim _{m \rightarrow 1}\left(b^{2} h^{2}\right)(m)=0 \quad \text { and } \quad \lim _{m \rightarrow 1}\left(b h^{2}\right)(m)=0 .
$$

Proof. Let $u(m):=(b h)^{2}(m)$. Then, taking the square of the both sides of (25) (iii), we have

$$
64 u^{3}+32(2 m-1) u^{2}+4 u=p^{2} h^{-6} .
$$

When $u \geq 0$ and $m \geq 1 / 2$, it holds that

$$
4 u \leq 64 u^{3}+32(2 m-1) u^{2}+4 u=p^{2} h^{-6},
$$

and hence we obtain (39). Since $h=4 n K(m) / L$ and $\lim _{m \rightarrow 1} K(m)=\infty$, we have (40). 
Using this lemma, we obtain:

Lemma 13.

$$
\lim _{m \rightarrow 1} F(m)=0 \text {. }
$$

Proof. Put $l=-a^{2} /\left(b^{2}-a^{2}\right)$. Since

$$
\begin{aligned}
\frac{b}{b^{2}-a^{2}} & =\frac{8 b h^{2}(1-m)}{1+4 b^{2} h^{2}-\sqrt{\left(1+4 b^{2} h^{2}\right)^{2}-16 b^{2} h^{2}(1-m)}} \\
& =\frac{1+4 b^{2} h^{2}+\sqrt{\left(1+4 b^{2} h^{2}\right)^{2}-16 b^{2} h^{2}(1-m)}}{2 b}>0
\end{aligned}
$$

and

$$
\frac{1}{1-l} \leq \frac{\Pi(l, m)}{K(m)} \leq 1
$$

we have from (37)

$$
\frac{b}{b^{2}-a^{2}}\left(\frac{1}{1-l}+\frac{\left(b^{2}-a^{2}\right) c}{b}\right) \leq \frac{2 n}{L} F(m) \leq \frac{b}{b^{2}-a^{2}}\left(1+\frac{\left(b^{2}-a^{2}\right) c}{b}\right) .
$$

Letting $m \rightarrow 1$, we see that

$$
\begin{aligned}
\lim _{m \rightarrow 1} \frac{b}{b^{2}-a^{2}}\left(\frac{l}{1-l}+\left(1+\frac{\left(b^{2}-a^{2}\right) c}{b}\right)\right) \\
=\lim _{m \rightarrow 1} \frac{1+4 b^{2} h^{2}+\sqrt{\left(1+4 b^{2} h^{2}\right)^{2}-16 b^{2} h^{2}(1-m)}}{2 b} \\
\quad \times\left(\frac{l}{1-l}+\frac{4 b^{2} h^{2}}{1+4 b^{2} h^{2}+\sqrt{\left(1+4 b^{2} h^{2}\right)^{2}-16 b^{2} h^{2}(1-m)}}\right) \\
=\lim _{m \rightarrow 1} \frac{1+4 b^{2} h^{2}+\sqrt{\left(1+4 b^{2} h^{2}\right)^{2}-16 b^{2} h^{2}(1-m)}}{2} \\
\quad \times\left(\frac{1}{1-l} \cdot \frac{l}{b}+\frac{4 b h^{2}}{1+4 b^{2} h^{2}+\sqrt{\left(1+4 b^{2} h^{2}\right)^{2}-16 b^{2} h^{2}(1-m)}}\right) .
\end{aligned}
$$

On the other hand, we have

$$
\begin{aligned}
l & =\frac{1+4 b^{2} h^{2}(2 m-1)-\sqrt{\left(1-4 b^{2} h^{2}\right)^{2}+16 b^{2} h^{2} m}}{-1-4 b^{2} h^{2}+\sqrt{\left(1-4 b^{2} h^{2}\right)^{2}+16 b^{2} h^{2} m}} \\
& =\frac{1}{2}\left(-1+4 b^{2} h^{2}+\sqrt{\left(1-4 b^{2} h^{2}\right)^{2}+16 b^{2} h^{2} m}\right) \\
& =\frac{8 b^{2} h^{2} m}{1-4 b^{2} h^{2}+\sqrt{\left(1-4 b^{2} h^{2}\right)^{2}+16 b^{2} h^{2} m}} .
\end{aligned}
$$

Thus, by (40), $\lim _{m \rightarrow 1} l(m)=0$ and $\lim _{m \rightarrow 1}(l / b)(m)=0$. Therefore, the left hand side of the inequality of (42) tends to 0 as $m \rightarrow 1$. Substituting $l=0$ to the left 
hand side of (42), we have the right hand side. Hence, we can conclude that the right hand side of (42) also tends to 0 . This completes the proof.

The following formula is elementary.

Lemma 14. Assume $b>a \geq 0$. Then

$$
\int_{0}^{\frac{L}{2 n}}\left(\frac{1}{a \cos \left(\frac{2 n \pi s}{L}\right)+b}+c\right) d s=\frac{L}{2 n}\left(\frac{1}{\sqrt{b^{2}-a^{2}}}+c\right) .
$$

The next lemma studies the behavior of $F(0)$.

LEMMA $15 . \quad F(0)$ is

$$
\left\{\begin{array}{l}
\text { triple-valued, } \quad \text { if } 0<p<\frac{16 \pi^{3} n^{3}}{3 \sqrt{3} L^{3}}, \\
\text { double-valued, } \quad \text { if } p=\frac{16 \pi^{3} n^{3}}{3 \sqrt{3} L^{3}}, \\
\text { single-valued, } \quad \text { if } \frac{16 \pi^{3} n^{3}}{3 \sqrt{3} L^{3}}<p .
\end{array}\right.
$$

Proof. Substituting $m=0$ to (25) (i), (ii), (iii), and putting $v:=b h$, we obtain if $1-4 v^{2}>0$,

$$
\begin{aligned}
& a=-\sqrt{\frac{-1+4 v^{2}+\sqrt{\left(1-4 v^{2}\right)^{2}}}{8 h^{2}}}=0, \\
& c=-\frac{1+\sqrt{\left(1-4 v^{2}\right)^{2}}}{2 b}=-\frac{1-2 v^{2}}{b}, \\
& \frac{16 \pi^{3} n^{3}}{L^{3}} v\left(1-4 v^{2}\right)=p .
\end{aligned}
$$

Therefore, by Lemma 14,

$$
F(0)=\frac{L}{2 n}\left(\frac{1}{b}-\frac{1-2 v^{2}}{b}\right)=\frac{L v^{2}}{n b}=2 \pi v,
$$

because in this case $h=4 n K(0) / L=2 n \pi / L$. The graph of the function $f(v):=$ $\left(16 \pi^{3} n^{3} / L^{3}\right) v\left(1-4 v^{2}\right)$ is plotted in Fig. 4 (i). Hence the graph of $p$ versus $F(0)$ looks like Fig. 4 (ii). If $1-4 v^{2} \leq 0$, again by substituting $m=0$ to (25) (i), (ii), (iii), we have

$$
\begin{aligned}
& a=-\frac{\sqrt{4 v^{2}-1}}{2 h}, \\
& c=-\frac{1+\left(4 v^{2}-1\right)}{2 b}=-\frac{2 v^{2}}{b}=-2 v h, \\
& \frac{16 \pi^{3} n^{3}}{L^{3}} v\left(4 v^{2}-1\right)=p .
\end{aligned}
$$


Thus, $b^{2}-a^{2}=b^{2}-\left(4 v^{2}-1\right) /\left(4 h^{2}\right)=1 /\left(4 h^{2}\right)$, and hence $F(0)=(L / 2 n)(2 h-2 v h)=$ $2 \pi(1-v)$. The graph of the function $f(v):=\left(16 \pi^{3} n^{3} / L^{3}\right) v\left(4 v^{2}-1\right)$ looks like Fig. 5 (i), therefore the graph $p$ versus $F(0)$ is as in Fig. 5 (ii).
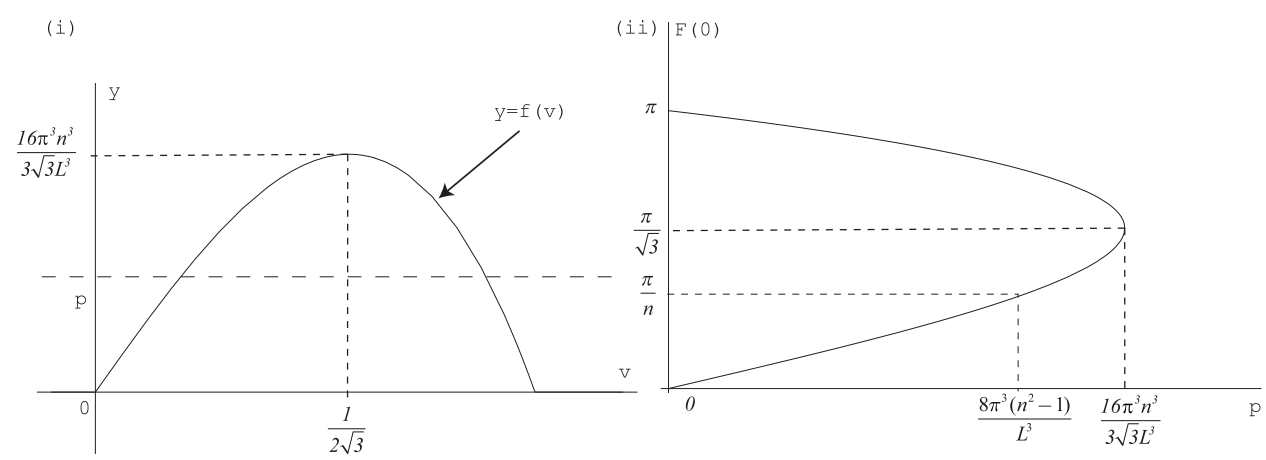

Fig. 4. The graphs of functions $v$ versus $f(v)$ and $p$ versus $F(0)$ when $1-4 v^{2}>0$.
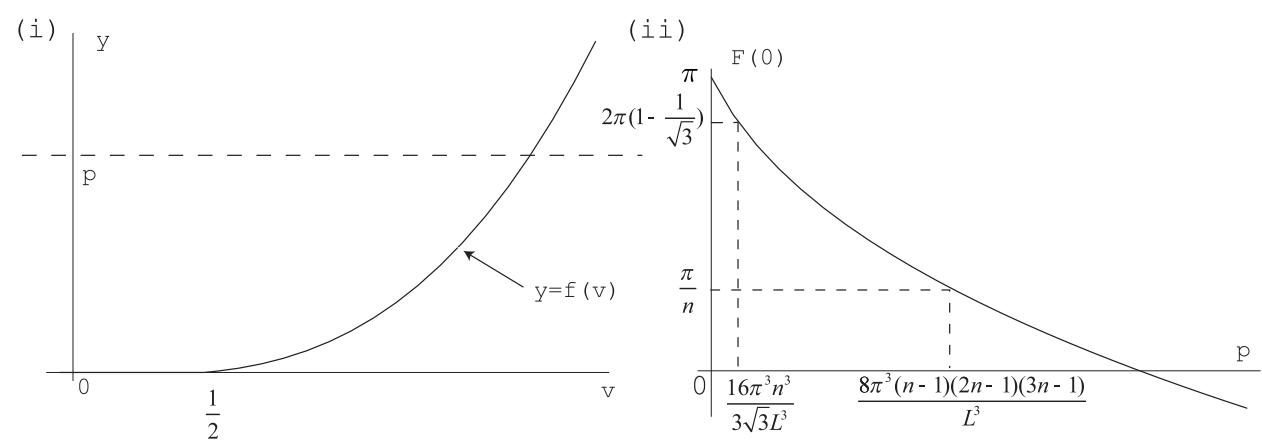

Fig. 5. The graphs of the functions $v$ versus $f(v)$ and $p$ versus $F(0)$ when $1-4 v^{2} \leq 0$.

Definition 3. Let us denote by $F_{1}(0), F_{2}(0)$ and $F_{3}(0)$ the value of $F(0)$ for fixed $p$, according to the following three cases:

$$
\left\{\begin{array}{l}
F_{1}(0), \quad \text { if } 0<p \leq 16 \pi^{3} n^{3} / 3 \sqrt{3} L^{3}, 1-4 v^{2}>0 \text { and } F(0) \leq \pi / \sqrt{3} \\
F_{2}(0), \quad \text { if } 0<p \leq 16 \pi^{3} n^{3} / 3 \sqrt{3} L^{3}, 1-4 v^{2}>0 \text { and } F(0)>\pi / \sqrt{3} \\
F_{3}(0), \quad \text { if } 0<p, 1-4 v^{2} \leq 0
\end{array}\right.
$$


COROLlary 2. From Figs. 4 and 5 we have the following relations for $F_{1}(0)$, $F_{2}(0)$ and $F_{3}(0)$.

$$
\begin{aligned}
& \text { (i) } \quad F_{1}(0)<F_{2}(0), \quad \text { if } 0<p<\frac{16 \pi^{3} n^{3}}{3 \sqrt{3} L^{3}}, \\
& \text { (ii) } \quad F_{1}(0)=F_{2}(0), \quad \text { if } p=\frac{16 \pi^{3} n^{3}}{3 \sqrt{3} L^{3}}, \\
& \text { (iii) } \quad F_{1}(0)<\frac{\pi}{n}<F_{2}(0) \text {, if } p<\frac{8 \pi^{3}\left(n^{2}-1\right)}{L^{3}}, n \geq 2 \text {, } \\
& \text { (iv) } \frac{\pi}{n}=F_{1}(0)<F_{2}(0) \text {, if } p=\frac{8 \pi^{3}\left(n^{2}-1\right)}{L^{3}}, n \geq 2 \text {, } \\
& \text { (v) } \frac{\pi}{n}<F_{1}(0) \leq F_{2}(0), \quad \text { if } \frac{8 \pi^{3}\left(n^{2}-1\right)}{L^{3}}<p \leq \frac{16 \pi^{3} n^{3}}{3 \sqrt{3} L^{3}}, n \geq 2 \text {, } \\
& \text { (vi) } \quad F_{3}(0)>\frac{\pi}{n}, \quad \text { if } p<\frac{8 \pi^{3}(n-1)(2 n-1)(3 n-1)}{L^{3}} \text {, } \\
& \text { (vii) } \quad F_{3}(0)=\frac{\pi}{n}, \quad \text { if } p=\frac{8 \pi^{3}(n-1)(2 n-1)(3 n-1)}{L^{3}} \text {, } \\
& \text { (viii) } \quad F_{3}(0)<\frac{\pi}{n}, \quad \text { if } \frac{8 \pi^{3}(n-1)(2 n-1)(3 n-1)}{L^{3}}<p \text {. }
\end{aligned}
$$

Proof. Assertions (i) and (ii) are obvious. When $2 \pi v=\pi / n$, we have $v=$ $1 /(2 n)$, so $p=2 v\left(1-4 v^{2}\right)(2 n \pi / L)^{3}=8 \pi^{3}\left(n^{2}-1\right) / L^{3}$. Since $F_{1}(0)$ is monotone increasing we have (iii), (iv) and (v). If $2 \pi(1-v)=\pi / n$, we have $v=1-1 /(2 n)$, so $p=2 v\left(4 v^{2}-1\right)(2 n \pi / L)^{3}=8 \pi^{3}(n-1)(2 n-1)(3 n-1) / L^{3}$. Since $F_{3}(0)$ is monotone decreasing we have (vi), (vii) and (viii).

We prepare differentiation formulas for complete elliptic integrals.

Lemma 16 (Differentiation formulas). The following formulas hold:

$$
\begin{aligned}
\frac{\partial \Pi}{\partial l}(l, m) & =\frac{l E(m)+(m-l) K(m)+\left(l^{2}-m\right) \Pi(l, m)}{2 l(1-l)(l-m)}, \\
\frac{\partial \Pi}{\partial m}(l, m) & =\frac{E(m)-(1-m) \Pi(l, m)}{2(l-m)(-1+m)} \\
\frac{d K}{d m}(m) & =\frac{E(m)-(1-m) K(m)}{2 m(1-m)}, \\
\frac{d E}{d m}(m) & =\frac{E(m)-K(m)}{2 m}
\end{aligned}
$$

where $E(\cdot)$ is the complete elliptic integral of the second kind ([1, p. 590]).

Proof. For (53) and (54), see Whittaker and Watson [17, p. 521]. For (51) and (52), see [18]. 
By making use of (51) and (52), we obtain the following lemma.

LEMma 17. Let u satisfy (41). Then

$$
\frac{d F}{d m}(m)=\frac{-L^{6} p^{2}\left\{3 E^{2}(m)+(-5+4 m) E(m) K(m)+2(1-m) K^{2}(m)\right\}}{2^{12} m(1-m) n^{6} K^{7}(m) \sqrt{u}\left\{48 u^{2}+16(2 m-1) u+1\right\} \sqrt{(1-4 u)^{2}+16 m u}} .
$$

Proof. Substituting $b=u^{\frac{1}{2}} / h$ to (25), we have

$$
\begin{aligned}
& a=-\frac{L}{8 \sqrt{2} n K(m)} \sqrt{\frac{-1+4(1-2 m) u+\sqrt{(1-4 u)^{2}+16 m u}}{1-m}}, \\
& c=-\frac{2 n K(m)\left(1+\sqrt{(1-4 u)^{2}+16 m u}\right)}{L u^{\frac{1}{2}}} .
\end{aligned}
$$

Using (56), (57) and Lemma 16, we have

$$
\begin{aligned}
\frac{d F(m)}{d m}= & \frac{2}{(1-m) m u^{\frac{1}{2}} \sqrt{(1-4 u)^{2}+16 m u}} \\
& \times\left\{((1-2 m) E(m)-(1-m) K(m)) u+(-4 E(m)+4(1-m) K(m)) u^{2}\right. \\
& \left.\quad+(2 m(1-m) E(m)-m(1-m) K(m)-4 m(1-m) K(m) u) \frac{d u(m)}{d m}\right\} .
\end{aligned}
$$

By differentiating both sides of (41) with respect to $m$, we obtain

$$
\frac{d u(m)}{d m}=\frac{\frac{-3 L^{6} p^{2}(E(m)-(1-m) K(m))}{2^{14} m(1-m) n^{6} K^{7}(m)}-16 u^{2}}{48 u^{2}+16(2 m-1) u+1} .
$$

Substituting this into (58) and eliminating $u^{3}$ and $u^{4}$ by using the relations

$$
\begin{aligned}
& u^{3}=\frac{1}{64}\left(\frac{p^{2}}{h^{6}}-4 u+32(1-2 m) u^{2}\right) \\
& u^{4}=\frac{1}{128 h^{6}}\left\{(1-2 m) p^{2}+2\left(-2 h^{6}(1-2 m)+p^{2}\right) u+8 h^{6}\left(3-16 m+16 m^{2}\right) u^{2}\right\}
\end{aligned}
$$

and after lengthy (but elementary) computations, we obtain the result.

To determine the sign of $d F(m) / d m$, we prepare the following two lemmas.

LEMMA 18. If $m \neq 0$, then

$$
(-1+2 m) E(m)+(1-m) K(m)>0 .
$$


Proof. Noting that

$$
\begin{aligned}
(1-m) K(m) & =\int_{0}^{1} \frac{1-m x^{2}-m+m x^{2}}{\sqrt{\left(1-x^{2}\right)\left(1-m x^{2}\right)}} d x \\
& =E(m)-m \int_{0}^{1} \sqrt{\frac{1-x^{2}}{1-m x^{2}}} d x
\end{aligned}
$$

we obtain

$$
\begin{aligned}
& (-1+2 m) E(m)+(1-m) K(m) \\
& =m \int_{0}^{1} 2 \sqrt{\frac{1-m x^{2}}{1-x^{2}}}-\sqrt{\frac{1-x^{2}}{1-m x^{2}}} d x=m \int_{0}^{1} \frac{1+(1-2 m) x^{2}}{\sqrt{\left(1-x^{2}\right)\left(1-m x^{2}\right)}} d x \\
& >m \int_{0}^{1} \frac{1-x^{2}}{\sqrt{\left(1-x^{2}\right)\left(1-m x^{2}\right)}} d x=m \int_{0}^{1} \sqrt{\frac{1-x^{2}}{1-m x^{2}}} d x>0
\end{aligned}
$$

which is positive, if $m \neq 0$.

Lemma 19. Put

$$
G(m):=3 E^{2}(m)+(-5+4 m) E(m) K(m)+2(1-m) K^{2}(m) .
$$

Then there exists $m_{z}\left(0<m_{z}<1\right)$, such that

$$
\left\{\begin{array}{l}
G(m)>0 \quad \text { if } 0<m<m_{z} \\
G(m) \leq 0 \quad \text { if } m_{z} \leq m<1
\end{array}\right.
$$

Proof. Using (53) and (54), we have

$$
\begin{aligned}
\frac{d G}{d m}(m) & =\frac{1}{2 m(1-m)}\left\{(1-2 m) E^{2}(m)-2(1-m)(1-4 m) E(m) K(m)\right. \\
\left.+(1-m)(1-4 m) K^{2}(m)\right\}, & \\
\frac{d^{2} G}{d m^{2}}(m) & =-\frac{((-1+2 m) E(m)+(1-m) K(m))^{2}}{2 m^{2}(1-m)^{2}}<0 \quad(\text { if } m \neq 0) .
\end{aligned}
$$

Applying l'Hospital's rule, we obtain also that

$$
\lim _{m \rightarrow 0} \frac{d G}{d m}(m)=\lim _{m \rightarrow 0} \frac{K(m)(8(1-2 m) E(m)-(5-8 m) K(m))}{2-4 m}=\frac{3 \pi^{2}}{8},
$$

and

$$
\lim _{m \rightarrow 1} \frac{d G}{d m}(m)=-\infty .
$$

Therefore there exists $m_{c} \in(0,1)$ such that $d G(m) / d m$ is positive if $0 \leq m<m_{c}$ and negative if $m_{c}<m<1$. Since $K(0)=E(0)=\pi / 2$, we have $G(0)=0$. It is known that $K(m) \sim \log (1 \sqrt{1-m})$ as $m \rightarrow 1$ [1, p. 591], and $E(1)=1$, hence $G(1)=-\infty$. Consequently $G(m)$ changes its sign exactly once in the interval $0<m<1$. 
In order to know the sign of $d F(m) / d m$, we need to investigate the sign of $48 u^{2}+16(2 m-1) u+1$ which appears in the denominator of $(55)$. Let us now define a function $\varphi$ by

$$
\varphi(u):=64 u^{3}+32(2 m-1) u^{2}+4 u-\frac{p^{2}}{h^{6}(m)} .
$$

Then, the equation (41) can be rewritten as $\varphi(u)=0$.

Lemma 20. The quadratic equation $\varphi^{\prime}(u)=0$ has two positive roots $\alpha$ and $\beta(0<\alpha \leq \beta)$, if and only if $0 \leq m \leq(2-\sqrt{3}) / 4$. Moreover, let $f_{0}(m):=$ $\sqrt{h^{6}(m) \varphi(\alpha)+p^{2}}$ and $f_{1}(m):=\sqrt{h^{6}(m) \varphi(\beta)+p^{2}}$. Then $f_{0}$ and $f_{1}$ behave as in Fig. 6. In particular, it holds that

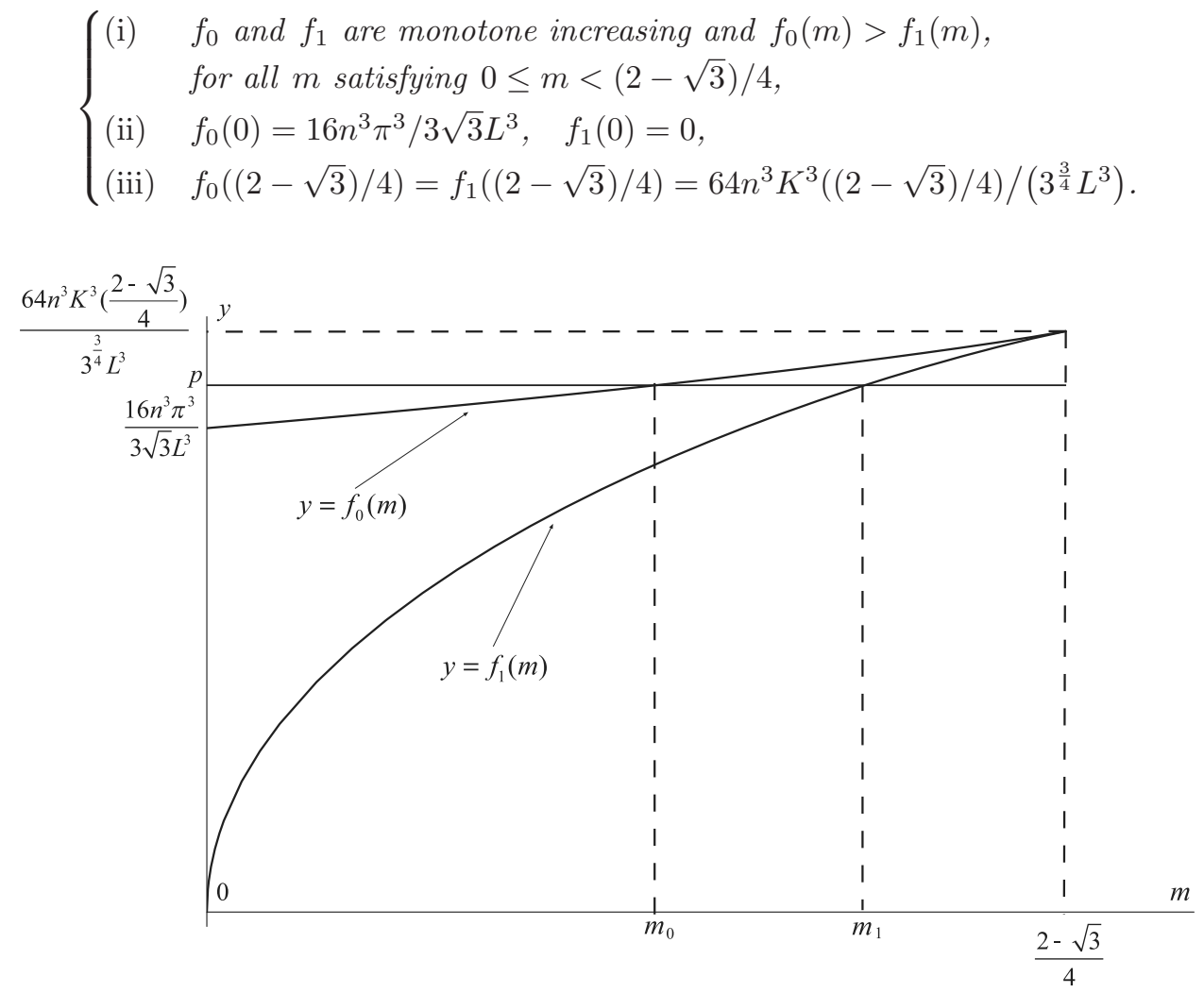

Fig. 6. $y=f_{0}(m)$ and $y=f_{1}(m)$.

Proof. Substituting $\alpha$ and $\beta$ in $\varphi$, we obtain

$$
\begin{aligned}
& f_{0}(m)=h^{3}(m)\left\{\frac{2}{27}\left(64 m^{3}-96 m^{2}+30 m+1+\left(16 m^{2}-16 m+1\right)^{\frac{3}{2}}\right)\right\}^{\frac{1}{2}}, \\
& f_{1}(m)=h^{3}(m)\left\{\frac{2}{27}\left(64 m^{3}-96 m^{2}+30 m+1-\left(16 m^{2}-16 m+1\right)^{\frac{3}{2}}\right)\right\}^{\frac{1}{2}} .
\end{aligned}
$$


It is easy to check the monotonicity of $f_{0}$ and $f_{1}$. Moreover, since $\varphi(\beta) \leq \varphi(\alpha)$ by definition, so $f_{1}(m) \leq f_{0}(m)$. The remaining parts are easy to verify.

The next lemma clarifies the sign of $48 u^{2}+16(2 m-1) u+1=\varphi^{\prime}(u) / 4$.

Lemma 21. Let $m_{0}, m_{1}$ be the solutions of $f_{0}(m)=p$, and $f_{1}(m)=p$, respectively (see Fig. 6) and let $u_{1}, u_{2}$ and $u_{3}$ be the roots of $\varphi(u)=0$ satisfying $u_{1} \leq \alpha \leq u_{2} \leq \beta \leq u_{3}$. Then the sign of $\varphi\left(u_{i}\right)(i=1,2,3)$ is as in Table 1 .

Table 1. Sign of $\varphi^{\prime}\left(u_{i}\right)$

\begin{tabular}{c|c|c}
\hline$p$ & $m$ & Sign of $\varphi^{\prime}\left(u_{i}\right)$ \\
\hline $0 \leq p<\frac{16 n^{3} \pi^{3}}{3 \sqrt{3} L^{3}}$ & $0 \leq m \leq m_{1}$ & $\varphi^{\prime}\left(u_{1}\right)>0, \varphi^{\prime}\left(u_{2}\right)<0, \varphi^{\prime}\left(u_{3}\right) \geq 0$ \\
\cline { 2 - 3 } & $m_{1}<m \leq 1$ & $\varphi^{\prime}\left(u_{1}\right)>0, u_{2}, u_{3}$ : not exist \\
\hline$\frac{16 n^{3} \pi^{3}}{3 \sqrt{3} L^{3}} \leq p<\frac{64 n^{3} K^{3}\left(\frac{2-\sqrt{3}}{4}\right)}{3^{\frac{3}{4}} L^{3}}$ & $0 \leq m<m_{0}$ & $\varphi^{\prime}\left(u_{3}\right)>0, u_{1}, u_{2}$ : not exist \\
\cline { 2 - 3 } & $m_{0} \leq m \leq m_{1}$ & $\varphi^{\prime}\left(u_{1}\right) \geq 0, \varphi^{\prime}\left(u_{2}\right) \leq 0, \varphi^{\prime}\left(u_{3}\right) \geq 0$ \\
\cline { 2 - 3 } & $m_{1}<m \leq 1$ & $\varphi^{\prime}\left(u_{1}\right)>0, u_{2}, u_{3}$ : not exist \\
\hline$\frac{64 n^{3} K^{3}\left(\frac{2-\sqrt{3}}{4}\right)}{3^{\frac{3}{4}} L^{3}} \leq p$ & $0 \leq m \leq 1$ & $\varphi^{\prime}\left(u_{3}\right) \geq 0, u_{1}, u_{2}$ : not exist \\
\hline
\end{tabular}

Proof. We shall prove the case

$$
\left(16 n^{3} \pi^{3}\right) /\left(3 \sqrt{3} L^{3}\right) \leq p<\left(64 n^{3} K^{3}\left(\frac{2-\sqrt{3}}{4}\right)\right) /\left(3^{\frac{3}{4}} L^{3}\right)
$$

only, for the other cases are treated similarly. Since $\varphi(\alpha)=\left(f_{0}^{2}-p^{2}\right) / h^{6}$ and $\varphi(\beta)=\left(f_{1}^{2}-p^{2}\right) / h^{6}$, we see that $\varphi(\alpha)>0$ if and only if $p<f_{0}(m)$, while $\varphi(\beta)<0$ if and only if $p>f_{1}(m)$. Thus, we obtain:

(i) If $0 \leq m<m_{0}$, then $\varphi(\alpha)<0$, and hence $\varphi(u)=0$ has only one real root $u=u_{3}$ satisfying $\beta<u_{3}$. Therefore $\varphi^{\prime}\left(u_{3}\right)>0$; see Fig. 7 (i).

(ii) If $m_{0} \leq m \leq m_{1}$, then $\varphi(\alpha) \geq 0, \varphi(\beta) \leq 0$. Hence $\varphi(u)=0$ has three real roots; see Fig. 7 (ii). Therefore $\varphi^{\prime}\left(u_{1}\right) \geq 0, \varphi^{\prime}\left(u_{2}\right) \leq 0, \varphi^{\prime}\left(u_{3}\right) \geq 0$. The equality holds when $m=m_{0}\left(u_{1}=u_{2}\right)$ or $m=m_{1}\left(u_{2}=u_{3}\right)$.

(iii) If $m_{1}<m$, then $\varphi(\beta)>0$, and hence $\varphi(u)=0$ has only one real root $u=u_{1}$ satisfying $u_{1}<\alpha$. Therefore $\varphi^{\prime}\left(u_{1}\right)>0$; see Fig. 7 (iii) and (iv).

The next lemma asserts that $m_{z}$ is greater than $m_{1}$.

Lemma 22. It holds that

$$
m_{0} \leq m_{1}<\frac{2-\sqrt{3}}{4}<m_{z} .
$$

Proof. Since $G((2-\sqrt{3}) / 4)>0.241>0$, from Lemma 19 we obtain the assertion. 


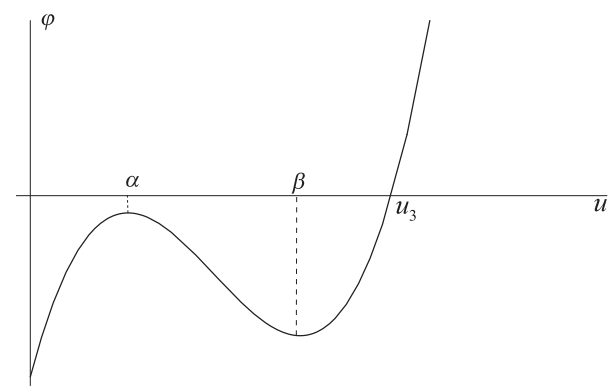

(i) $\left(0 \leq m<m_{0}\right)$

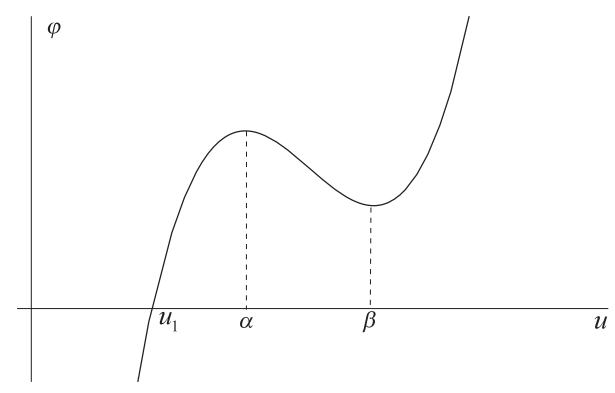

(iii) $\left(m_{1}<m<\frac{2-\sqrt{3}}{4}\right)$

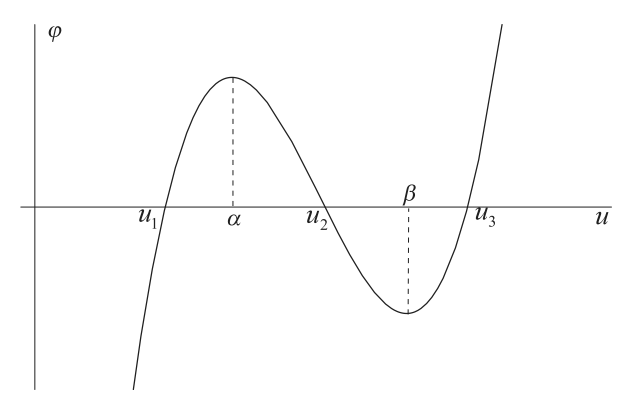

(ii) $\left(m_{0} \leq m \leq m_{1}\right)$

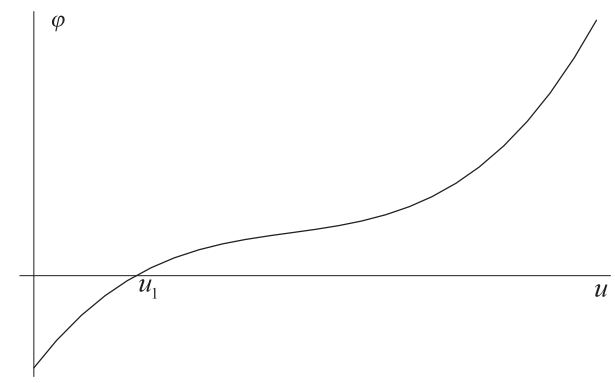

(iv) $\left(\frac{2-\sqrt{3}}{4} \leq m<1\right)$

Fig. 7. The graph of $\varphi$.

Lemma 23. Let $u_{1}, u_{2}, u_{3}$ be the roots of $\varphi(u)=0$, and let $\kappa_{i}(s)(i=1,2,3)$ be defined by (5), where

$$
b(m)=\sqrt{u_{i}(m)} / h(m),
$$

$a(m)$ and $c(m)$ are obtained by substituting (65) in (6) (i) and (6) (ii) respectively. Further, put

$$
F_{i}(m):=\int_{0}^{L /(2 n)} \kappa_{i}(s ; m) d s .
$$

Then, $y=F_{i}(m)(i=1,2,3)$ behaves as follows:
(i) in Fig. 8 (i) if $0<p<\frac{16 n^{3} \pi^{3}}{3 \sqrt{3} L^{3}}$,
(ii) in Fig. 8 (ii) if $\frac{16 n^{3} \pi^{3}}{3 \sqrt{3} L^{3}} \leq p<\frac{64 n^{3} K^{3}\left(\frac{2-\sqrt{3}}{4}\right)}{3^{\frac{3}{4}} L^{3}}$,
(iii) in Fig. 8 (iii) if $\frac{64 n^{3} K^{3}\left(\frac{2-\sqrt{3}}{4}\right)}{3^{\frac{3}{4}} L^{3}} \leq p$. 


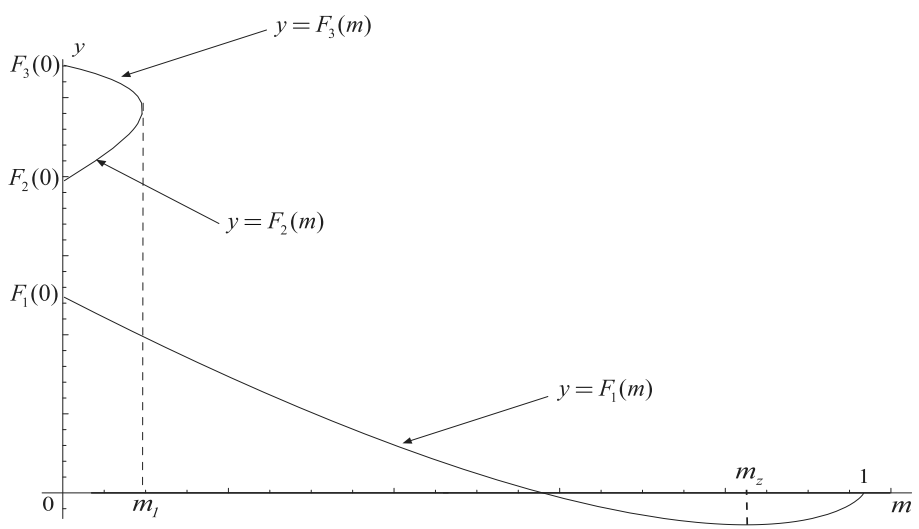

(i)

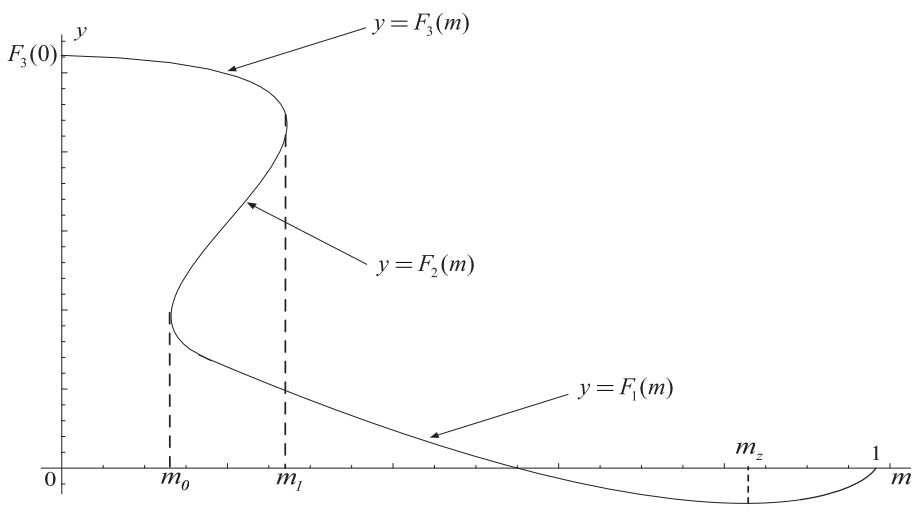

(ii)

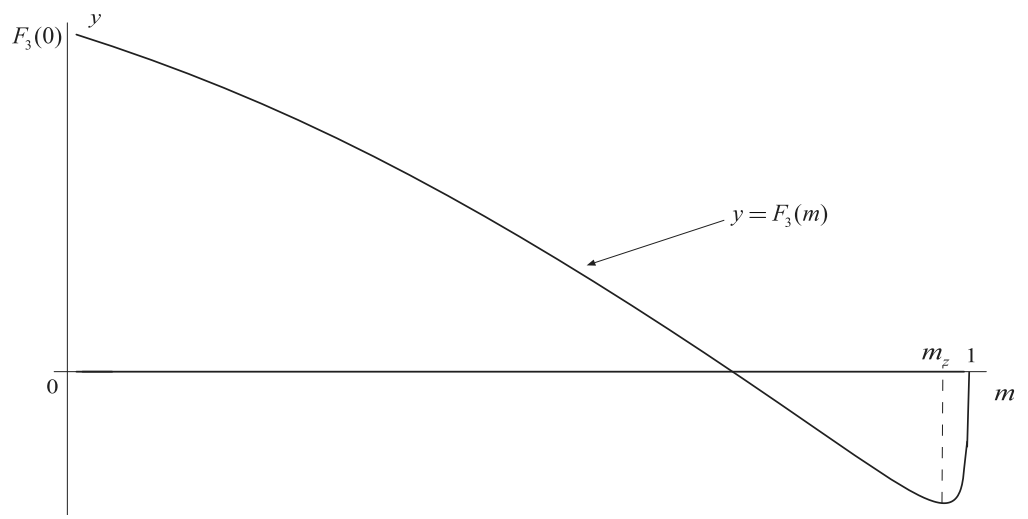

(iii)

Fig. 8. $y=F_{i}(m)(i=1,2,3)$ for various ranges of $p$. 
Proof. Let us consider Case (i). By Lemma 17 and Lemma 22, when $0 \leq m<$ $m_{z}$, the sign of $d F_{i}(m) / d m$ is opposite to that of $\varphi^{\prime}\left(u_{i}(m)\right)(i=1,2,3)$. Noting this, we prove Case (i) in four steps:

(I) If $0 \leq m \leq m_{1}$, then by Lemma $21, F_{1}(m)$ and $F_{3}(m)$ are monotone decreasing and $F_{2}(m)$ is monotone increasing. Moreover, since $u_{2}\left(m_{1}\right)=u_{3}\left(m_{1}\right)$, we have $F_{2}\left(m_{1}\right)=F_{3}\left(m_{1}\right)$, and hence the graphs $y=F_{2}(m)$ and $y=F_{3}(m)$ are like Fig. 8 (i). Moreover, $y=F_{2}(m)$ does not intersect with $y=F_{1}(m)$ because $F_{1}(0)<F_{2}(0)$ by Corollary $2(\mathrm{i})$.

(II) If $m_{1}<m \leq m_{z}$, then it holds that $\varphi^{\prime}\left(u_{1}(m)\right)>0$ by Lemma 21 , so $F_{1}(m)$ is monotone decreasing.

(III) If $m_{z} \leq m$, then the sign of $d F_{1}(m) / d m$ is the same as $\varphi^{\prime}\left(u_{1}(m)\right)$, because $G(m) \leq 0$. Therefore, $F_{1}(m)$ is monotone increasing.

(IV) From Lemma 13, $\lim _{m \rightarrow 1} F_{1}(m)=0$.

Summarizing the arguments (I)-(IV), as a consequence, we obtain the result. Cases (ii) and (iii) can be proven in the same way.

Now we are in a position to prove the first half of Theorem 2.

Proposition 1. Let $n$ be an arbitrary integer with $n \geq 2$. Then a necessary and sufficient condition for the equations (12)-(14) to have a nontrivial solution of the form (19) (i) is

$$
\frac{8 \pi^{3}}{L^{3}}\left(n^{2}-1\right)<p \leq \frac{8 \pi^{3}}{L^{3}}(n-1)(2 n-1)(3 n-1) .
$$

If $n=1$, then (12)-(14) does not have any nontrivial solution of the form (19) (i).

Proof. Note that if $n \geq 2$, it holds that

$$
\begin{aligned}
& \frac{8 \pi^{3}\left(n^{2}-1\right)}{L^{3}}<\frac{16 \pi^{3} n^{3}}{3 \sqrt{3} L^{3}}, \\
& \frac{64 n^{3} K^{3}\left(\frac{2-\sqrt{3}}{2}\right)}{3^{\frac{3}{4}} L^{3}}<\frac{8 \pi^{3}(n-1)(2 n-1)(3 n-1)}{L^{3}} .
\end{aligned}
$$

(I) If $0<p<8 \pi^{3}\left(n^{2}-1\right) / L^{3}$, then by (67) and Lemma 23 (i), the graphs of $y=F_{i}(m)(i=1,2,3)$ are as in Fig. 8 (i). On the other hand, by Corollary 2 (iii), $F_{1}(0)<\pi / n<F_{2}(0)$, hence neither $y=F_{1}(m)$ nor $y=F_{2}(m)$ intersects with $F=$ $\pi / n$. Therefore, in this case, no solution of the form (19) (i) exists.

(II) If $p=8 \pi^{3}\left(n^{2}-1\right) / L^{3}$, then $F_{1}(0)=\pi / n$. However, by a result in the proof of Lemma 15 , we see that $a(0)=0$, so (19) (i) does not present a nontrivial solution.

(III) If $8 \pi^{3}\left(n^{2}-1\right) / L^{3}<p<16 \pi^{3} n^{3} /\left(3 \sqrt{3} L^{3}\right)$, then by Lemma 23 (i), the graphs of $y=F_{i}(m)(i=1,2,3)$ are as in Fig. 8 (i). By Corollary $2(\mathrm{v}), F_{1}(0)>$ $\pi / n$, so there exists a unique $m^{*}$ satisfying $F_{1}\left(m^{*}\right)=\pi / n$. From Lemma 10 , we see that $a\left(m^{*}\right) \neq 0$. Therefore, in this case a nontrivial solution of the form (19) (i) uniquely exists. 
(IV) If $16 \pi^{3} n^{3} /\left(3 \sqrt{3} L^{3}\right) \leq p<8 \pi^{3}(n-1)(2 n-1)(3 n-1) / L^{3}$, then the graphs of $y=F_{i}(m)(i=1,2,3)$ are as in Fig. 8 (ii) or (iii). Since $F_{3}(0)>\pi / n$ by Corollary $2(\mathrm{vi})$, there exists a unique $m^{*}$ and $i(i \in\{1,2,3\})$ such that $F_{i}\left(m^{*}\right)=\pi / n$.

(V) If $p=8 \pi^{3}(n-1)(2 n-1)(3 n-1) / L^{3}$, then the graph of $y=F_{3}(m)$ is as in Fig. 8 (iii). From Corollary 2 (vii), $F_{3}(0)=\pi / n$. However, in this case $a(0)=$ $-L \sqrt{(n-1)(3 n-1)} /\left(4 n^{2} \pi\right) \neq 0$, so there exists a unique nontrivial solution of the form

$$
\kappa_{3}(s)=\frac{4 n^{2} \pi}{L\left(-\sqrt{(n-1)(3 n-1)} \cos \left(\frac{2 n \pi s}{L}\right)+(2 n-1)\right)}-\frac{2 \pi(2 n-1)}{L} .
$$

(VI) If $8 \pi^{3}(n-1)(2 n-1)(3 n-1) / L^{3}<p$, then by (68) and Lemma 23 (iii), the graph of $y=F_{3}(m)$ is like Fig. 8 (iii). Since by Corollary 2 (vii), $F_{3}(0)<\pi / n$, so $y=F_{3}(m)$ does not intersect with $F=\pi / n$. Therefore, in this case, no solution of the form (19) (i) exists.

When $n=1$, from Figs. 4 (ii) and 5 (ii), we see that for $i=1,2,3, F_{i}(0)<\pi$ hold when $p>0$, and hence for $i=1,2,3, F_{i}(m)=\pi$ does not have any solution. This completes the proof.

\subsection{Case of Lemma 7 (ii)}

In this subsection, we consider the case where $\kappa(s)$ is of the form (19) (ii).

LEMMA 24. Let

$$
\kappa(s)=\frac{1}{a \operatorname{dn}(h s)+b}+c \quad \text { with } h=\frac{2 n K(m)}{L}
$$

satisfies (12) and (13). Then $a, b$ and c satisfy either

$$
\left\{\begin{array}{l}
\text { (i) } a= \pm \sqrt{\frac{1+4 b^{2} h^{2}(2-m)-\sqrt{1+8 b^{2} h^{2}(2-m)+16 b^{4} h^{4} m^{2}}}{8 h^{2}(1-m)}}, \\
\text { (ii) } c=-\frac{1+\sqrt{1+8 b^{2} h^{2}(2-m)+16 b^{4} h^{4} m^{2}}}{2 b}, \\
\text { (iii) } 2 b h^{4} m^{2} \sqrt{1+8 b^{2} h^{2}(2-m)+16 b^{4} h^{4} m^{2}}=p,
\end{array}\right.
$$

or

$$
\left\{\begin{array}{l}
\text { (i) } a= \pm \sqrt{\frac{1+4 b^{2} h^{2}(2-m)+\sqrt{1+8 b^{2} h^{2}(2-m)+16 b^{4} h^{4} m^{2}}}{8 h^{2}(1-m)}}, \\
\text { (ii) } c=\frac{-1+\sqrt{1+8 b^{2} h^{2}(2-m)+16 b^{4} h^{4} m^{2}}}{2 b}, \\
\text { (iii) }-2 b h^{4} m^{2} \sqrt{1+8 b^{2} h^{2}(2-m)+16 b^{4} h^{4} m^{2}}=p .
\end{array}\right.
$$


Proof. This lemma can be proven in a way similar to that in the proof of Lemma 8.

The next lemma asserts that it is sufficient to consider only either of (69) or $(70)$.

Lemma 25. Let $\{a, b, c\}$ and $\left\{a^{\prime}, b^{\prime}, c^{\prime}\right\}$ satisfy (69) and (70) respectively. Then, it holds that

$$
\frac{1}{a \operatorname{dn}(h s+K(m))+b}+c=\frac{1}{a^{\prime} \operatorname{dn}(h s)+b^{\prime}}+c^{\prime},
$$

where the sign of $a^{\prime}$ is opposite to that of $a$.

Proof. Using the addition formula for $\operatorname{dn}(\cdot)$ [1, p. 574], we have $\operatorname{dn}(z+$ $K(m))=\sqrt{1-m} / \operatorname{dn}(z)$, so

$$
\frac{1}{a \operatorname{dn}(h s+K(m))+b}+c=\frac{1}{-\frac{b^{2}}{a \sqrt{1-m}} \operatorname{dn}(h s)-b}+\frac{1}{b}+c .
$$

Since

$$
\begin{aligned}
-\frac{b^{2}}{a \sqrt{1-m}} & =\mp \sqrt{\frac{8 b^{4} h^{2}}{1+4 b^{2} h^{2}(2-m)-\sqrt{1+8 b^{2} h^{2}(2-m)+16 b^{4} h^{4} m^{2}}}} \\
& =\mp \sqrt{\frac{1+4 b^{2} h^{2}(2-m)+\sqrt{1+8 b^{2} h^{2}(2-m)+16 b^{4} h^{4} m^{2}}}{8 h^{2}(1-m)}}=a^{\prime},
\end{aligned}
$$

$b^{\prime}=-b$, and

$$
\begin{aligned}
\frac{1}{b}+c & =\frac{1-\sqrt{1+8 b^{2} h^{2}(2-m)+16 b^{4} h^{4} m^{2}}}{2 b} \\
& =\frac{-1+\sqrt{1+8\left(b^{\prime}\right)^{2} h^{2}(2-m)+16\left(b^{\prime}\right)^{4} h^{4} m^{2}}}{2 b^{\prime}}=c^{\prime},
\end{aligned}
$$

we have proven the lemma.

Thus, from now on, we only consider the case (69).

Lemma 26. For any $p>0$ and $m$ satisfying $0<m<1$, (69) (iii) has a unique positive solution $b(m)$. we have

Proof. Taking the square of the both sides of (69) (iii), and putting $u:=(b h)^{2}$,

$$
4 m^{4} u\left(16 m^{2} u^{2}+8(2-m) u+1\right)=p^{2} h^{-6} .
$$

Let us denote the left hand side of $(71)$ by $f(u)$. Since $f$ is monotone increasing and $f(0)=0, f(\infty)=\infty,(71)$ has a unique positive solution. 
Definition 4. Assume $0<m<1$, and $b(m)$ be the unique solution of (69) (iii) stated in Lemma 26. We define the functions $H_{1}(m)$ and $H_{2}(m)$ as follows:

$$
\begin{aligned}
& H_{1}(m)=\int_{0}^{\frac{L}{2 n}}\left(\frac{1}{a^{(+)}(m) \operatorname{dn}\left(\frac{2 n K(m) s}{L}\right)+b(m)}+c(m)\right) d s, \\
& H_{2}(m)=\int_{0}^{\frac{L}{2 n}}\left(\frac{1}{a^{(-)}(m) \operatorname{dn}\left(\frac{2 n K(m) s}{L}\right)+b(m)}+c(m)\right) d s,
\end{aligned}
$$

where $a^{(+)}(m)$ is defined by

$$
a^{(+)}(m)=\sqrt{\frac{1+4 b^{2} h^{2}(2-m)-\sqrt{1+8 b^{2} h^{2}(2-m)+16 b^{4} h^{4} m^{2}}}{8 h^{2}(1-m)}}>0,
$$

and $a^{(-)}(m)=-a^{(+)}(m)$.

By Lemma 26, both $H_{1}(m)$ and $H_{2}(m)$ are single-valued functions. Before going to the study of $H_{i}(m)$, let us take a look at numerical examples to see how $H_{i}$ behave for various values of $p$. Fig. 9 shows the behavior of $H_{1}$ and $H_{2}$ for $n=2$, $p=14,15,16$ and $L=2 \pi$. From Fig. 9, we observe that equation $H_{1}(m)=\pi / 2$ seems to have no solution, while the equation $H_{2}(m)=\pi / 2$ seems to have

(i) no solution, if $p<15=8 \pi^{3}(n-1)(2 n-1)(3 n-1) / L^{3}$,

(ii) a unique solution, if $15 \leq p$.

To study the behavior of $H_{1}$ and $H_{2}$ near $m=1$, we need the following lemma.

Lemma 27. For the function $H_{1}(m)$, we have

$$
H_{1}(m)=-\pi+\frac{L b}{2 n\left(b^{2}-a^{2}\right)}\left(\frac{\Pi\left(-\frac{a^{2} m}{b^{2}-a^{2}}, m\right)}{K(m)}+\frac{\left(b^{2}-a^{2}\right) c}{b}\right)
$$

while for $H_{2}(m)$, we have

$$
H_{2}(m)=\pi+\frac{L b}{2 n\left(b^{2}-a^{2}\right)}\left(\frac{\Pi\left(-\frac{a^{2} m}{b^{2}-a^{2}}, m\right)}{K(m)}+\frac{\left(b^{2}-a^{2}\right) c}{b}\right) .
$$

Proof. Put $\operatorname{dn}(t)=x$. Then we have

$$
\begin{aligned}
\int_{0}^{\frac{L}{2 n}} \frac{1}{a \operatorname{dn}(h s)+b} d s & =\frac{1}{h} \int_{0}^{K(m)} \frac{1}{a \operatorname{dn}(t)+b} d t \\
& =\frac{1}{h} \int_{\sqrt{1-m}}^{1} \frac{1}{(a x+b) \sqrt{\left(1-x^{2}\right)\left(m-1+x^{2}\right)}} d x
\end{aligned}
$$




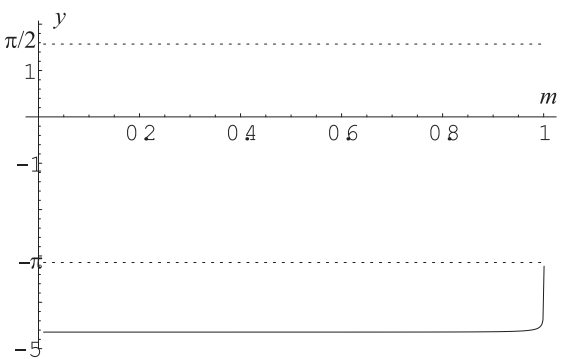

(i) $\mathrm{p}=14, y=H_{1}(m)$

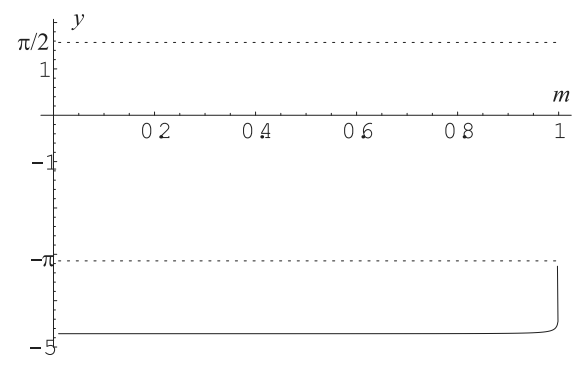

(iii) $\mathrm{p}=15, y=H_{1}(m)$

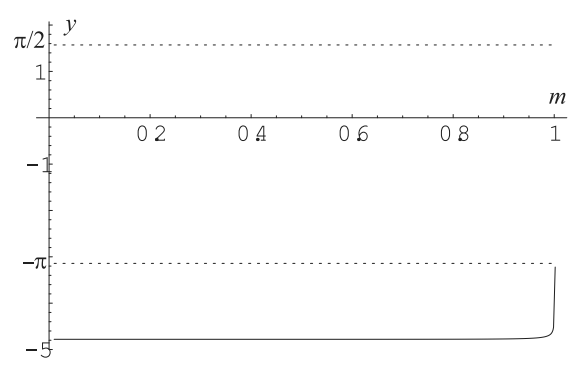

(v) $\mathrm{p}=16, y=H_{1}(m)$

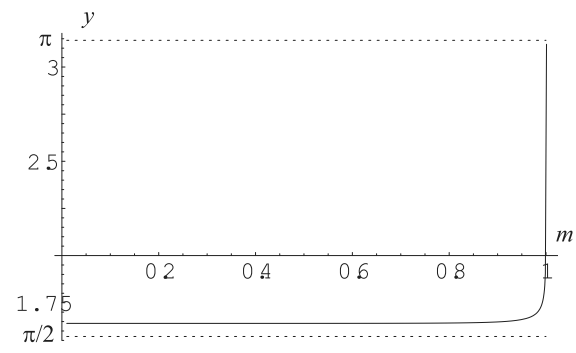

(ii) $\mathrm{p}=14, y=H_{2}(m)$

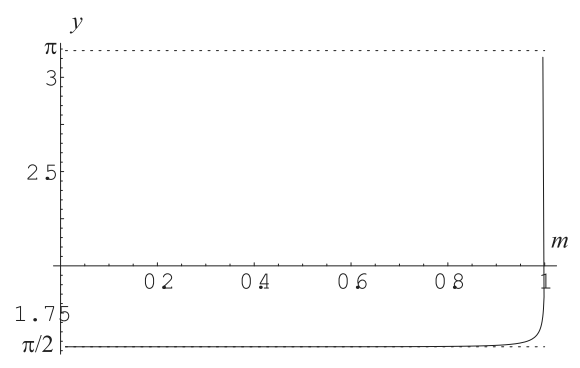

(iv) $\mathrm{p}=15, y=H_{2}(m)$

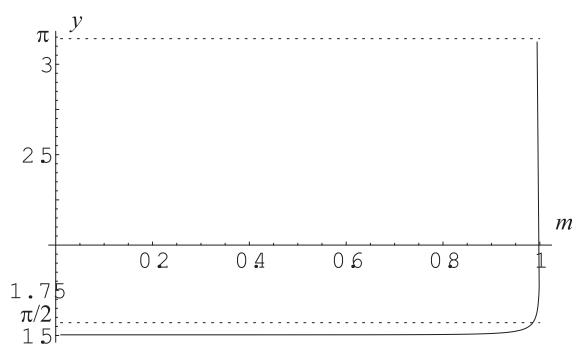

(vi) $\mathrm{p}=16, y=H_{2}(m)$

Fig. 9. The graphs of $y=H_{1}$ and $y=H_{2}$ for various $p$, when $n=2$ and $L=2 \pi$. 
and again putting $x^{2}=1-m z^{2}$, we obtain

$$
\begin{aligned}
& \frac{1}{h} \int_{0}^{1} \frac{1}{\left(a \sqrt{1-m z^{2}}+b\right) \sqrt{\left(1-z^{2}\right)\left(1-m z^{2}\right)}} d z \\
& =\frac{1}{h}\left(-a \int_{0}^{1} \frac{d z}{\left(b^{2}-a^{2}+a^{2} m z^{2}\right) \sqrt{1-z^{2}}}\right. \\
& \left.\quad+b \int_{0}^{1} \frac{d z}{\left(b^{2}-a^{2}+a^{2} m z^{2}\right) \sqrt{\left(1-z^{2}\right)\left(1-m z^{2}\right)}}\right) .
\end{aligned}
$$

Since

$$
b^{2}-a^{2}=\frac{-1-4 b^{2} h^{2} m+\sqrt{\left(1+4 b^{2} h^{2} m\right)^{2}+16 b^{2} h^{2}(1-m)}}{8 h^{2}(1-m)}>0,
$$

we can apply the calculus formula

$$
\int_{0}^{1} \frac{1}{\left(1+\alpha x^{2}\right) \sqrt{1-x^{2}}} d x=\frac{\pi}{2 \sqrt{1+\alpha}} \quad(\alpha>-1)
$$

and obtain

$$
\begin{aligned}
& \int_{0}^{\frac{L}{2 n}} \frac{1}{a \operatorname{dn}(h s)+b} d s \\
& =-\frac{a \pi}{2 h \sqrt{\left(b^{2}-a^{2}\right)\left(b^{2}-(1-m) a^{2}\right)}}+\frac{b}{h\left(b^{2}-a^{2}\right)} \Pi\left(-\frac{a^{2} m}{b^{2}-a^{2}}, m\right) .
\end{aligned}
$$

On the other hand,

$$
\begin{aligned}
& \left(b^{2}-a^{2}\right)\left(b^{2}-(1-m) a^{2}\right) \\
& =\frac{1+4 b^{2} h^{2}(2-m)-\sqrt{1+8 b^{2} h^{2}(2-m)+16 b^{4} h^{4} m^{2}}}{32 h^{4}(1-m)}=\frac{a^{2}}{4 h^{2}},
\end{aligned}
$$

so

$$
-\frac{a \pi}{2 h \sqrt{\left(b^{2}-a^{2}\right)\left(b^{2}-(1-m) a^{2}\right)}}= \begin{cases}-\pi, & a>0, \\ \pi, & a<0 .\end{cases}
$$

Substituting this equation to (78), we obtain the result.

Using Lemma 27 and the next lemma, we can estimate the behavior of $H_{1}$ and $H_{2}$ when $m$ approaches 1 .

Lemma 28. Let $b(m)$ be the solution of (69) (iii). Then the following estimate holds:

$$
b(m) \leq \frac{p}{2 m^{2} h^{4}(m)} .
$$


Proof. From (71), $4 m^{4} u \leq p^{2} / h^{6}$. Hence, we obtain the result.

Lemma 29. $\lim _{m \rightarrow 1} H_{1}(m)=-\pi$ and $\lim _{m \rightarrow 1} H_{2}(m)=\pi$.

Proof. Let

$$
J(m):=\frac{L b}{2 n\left(b^{2}-a^{2}\right)}\left(\frac{\Pi(l, m)}{K(m)}+\frac{\left(b^{2}-a^{2}\right) c}{b}\right),
$$

where $l=-a^{2} m /\left(b^{2}-a^{2}\right)$. Because of (76), we have the estimate

$$
\frac{b}{b^{2}-a^{2}}\left(\frac{1}{1-l}+\frac{\left(b^{2}-a^{2}\right) c}{b}\right) \leq \frac{2 n J(m)}{L} \leq \frac{b}{b^{2}-a^{2}}\left(1+\frac{\left(b^{2}-a^{2}\right) c}{b}\right) .
$$

The left hand side of (82) becomes

$$
\begin{aligned}
& \frac{b}{b^{2}-a^{2}}\left\{\frac{l}{1-l}+\left(1+\frac{\left(b^{2}-a^{2}\right) c}{b}\right)\right\} \\
& =\frac{1+4 b^{2} h^{2} m+\sqrt{\left(1+4 b^{2} h^{2} m\right)^{2}+16 b^{2} h^{2}(1-m)}}{2} \\
& \quad \times\left(\frac{1}{1-l} \cdot \frac{l}{b}+\frac{4 b h^{2} m}{1+4 b^{2} h^{2} m+\sqrt{\left(1+4 b^{2} h^{2} m\right)^{2}+16 b^{2} h^{2}(1-m)}}\right) .
\end{aligned}
$$

By Lemma 28,

$$
\lim _{m \rightarrow 1} l(m)=\lim _{m \rightarrow 1}-\frac{8 b^{2} h^{2} m}{1-4 b^{2} h^{2} m+\sqrt{\left(1-4 b^{2} h^{2} m\right)^{2}+16 b^{2} h^{2}}}=0,
$$

and $\lim _{m \rightarrow 1} \frac{l(m)}{b(m)}=0$. Therefore the left hand side of $(82)$ tends to 0 as $m \rightarrow 1$. The right hand side of (82) is obtained by substituting $l=0$ in the left hand side of (82). Hence the right hand side of (82) also converges to 0 as $m \rightarrow 1$.

The next lemma computes the derivative of $H_{2}$ (there is no need to compute the derivative of $H_{1}$ since $H_{1}=H_{2}-2 \pi$ by Lemma 27).

Lemma 30. Let u satisfy (71). Then the derivative of $\mathrm{H}_{2}$ is

$$
\frac{d H_{2}}{d m}(m)=\frac{L^{6} p^{2}\left(-3 E^{2}(m)+(2-m) E(m) K(m)+(1-m) K^{2}(m)\right)}{128 m^{5} n^{6} K^{7}(m)(1-m) u^{\frac{1}{2}} D(m)},
$$

where

$$
D(m)=\sqrt{1+8(2-m) u+16 m^{2} u^{2}}\left(1+16(2-m) u+48 m^{2} u^{2}\right) .
$$

Proof. This can be proven in the same way as in the proof of Lemma 17. 
To determine the sign of $d H_{2}(m) / d m$, we need the following two lemmas.

LEMMA 31. If $m \neq 0$,

$$
(-2+m) E(m)+2(1-m) K(m)<0 .
$$

Proof. Let

$$
G(m):=(-2+m) E(m)+2(1-m) K(m) .
$$

From (53) and (54), we have, if $m \neq 0$,

$$
\frac{d G}{d m}(m)=\frac{3}{2}(E(m)-K(m))<0 .
$$

Moreover, it holds that $G(0)=0$. Therefore, if $m \neq 0$, then $G(m)<0$.

LEMMA 32. Let

$$
I(m):=-3 E^{2}(m)+(2-m) E(m) K(m)+(1-m) K^{2}(m) .
$$

Then $I(m)>0$, if $m \neq 0$.

Proof. Using (53) and (54), we have

$$
\begin{aligned}
\frac{d I}{d m}(m) & =\frac{m^{2} E^{2}(m)-(1-m)(4-m)(K(m)-E(m))^{2}}{2 m(1-m)}, \\
\frac{d^{2} I}{d m^{2}}(m) & =\frac{((-2+m) E(m)+2(1-m) K(m))^{2}}{2 m^{2}(1-m)^{2}}
\end{aligned}
$$

By Lemma 31, if $m \neq 0, d^{2} I(m) / d m^{2}>0$. Moreover $(d I / d m)(0)=0$, hence $I$ is monotone increasing. Because $I(0)=0$, we have proven the lemma.

From Lemmas 30 and 32 , we see that $H_{i}(m)(i=1,2)$ is monotone increasing functions of $m$. Therefore, by Lemma 29, we see that the equation $H_{1}(m)=$ $\pi / n$ does not have any solution. Hence from now on, we consider the equation $H_{2}(m)=\pi / n$ only. As for the behavior of $H_{2}(m)$ when $m$ approaches 0 , we have the following lemma.

LEMMA 33.

$$
\lim _{m \rightarrow 0} H_{2}(m)\left\{\begin{array}{l}
>\frac{\pi}{n} \quad \text { if } p<\frac{8 \pi^{3}}{L^{3}}(n-1)(2 n-1)(3 n-1), \\
=\frac{\pi}{n} \quad \text { if } p=\frac{8 \pi^{3}}{L^{3}}(n-1)(2 n-1)(3 n-1), \\
<\frac{\pi}{n} \quad \text { if } p>\frac{8 \pi^{3}}{L^{3}}(n-1)(2 n-1)(3 n-1) .
\end{array}\right.
$$


Proof. From (69) (iii), when $m \rightarrow 0, b(m)$ seems to diverge with order $O(1 / m)$, so we put $b(m)=v(m) / m$ and substitute this to (69) (iii). This leads to the equation

$$
p=2 h^{4} v \sqrt{m^{2}+8 h^{2} v^{2}(2-m)+16 h^{4} v^{4}},
$$

and we see that (88) has a unique positive solution $v(m)$ for arbitrary $m$ satisfying $0 \leq m<1$ and $p>0$ (since the right hand side of (88) is monotone increasing in $v$ ). Now, we have proven that $b(m)$ diverges to $+\infty$ with the order of $1 / m$ when $m$ approaches 0 . Next, we estimate the function $\mathrm{H}_{2}$. Since

$$
b^{2}-a^{2}=\frac{-m-4 h^{2} v^{2}+\sqrt{16 h^{2} v^{2}\left(1+h^{2} v^{2}\right)-8 h^{2} v^{2} m+m^{2}}}{8 h^{2} m(1-m)},
$$

we have

$$
\lim _{m \rightarrow 0} \frac{b}{b^{2}-a^{2}}=\frac{8 h_{0}^{2} v_{0}}{-4 h_{0}^{2} v_{0}^{2}+4 h_{0} v_{0} \sqrt{1+h_{0}^{2} v_{0}^{2}}}=\frac{2 h_{0}}{-h_{0} v_{0}+\sqrt{1+h_{0}^{2} v_{0}^{2}}}
$$

and

$$
\lim _{m \rightarrow 0} \frac{a^{2} m}{b^{2}-a^{2}}=\frac{2 h_{0} v_{0}}{-h_{0} v_{0}+\sqrt{1+h_{0}^{2} v_{0}^{2}}}=2 h_{0} v_{0}\left(\sqrt{1+h_{0}^{2} v_{0}^{2}}+h_{0} v_{0}\right),
$$

where we have put $h_{0}=h(0)$ and $v_{0}=v(0)$ for simplicity. Using the formula (77) again, we obtain

$$
\lim _{m \rightarrow 0} \frac{\Pi\left(-\frac{a^{2} m}{b^{2}-a^{2}}, m\right)}{K(m)}=\frac{1}{\sqrt{2 h_{0}^{2} v_{0}^{2}+2 h_{0} v_{0} \sqrt{1+h_{0}^{2} v_{0}^{2}}+1}}=\frac{1}{h_{0} v_{0}+\sqrt{1+h_{0}^{2} v_{0}^{2}}} .
$$

Moreover we have

$$
\lim _{m \rightarrow 0} c(m)=-2 h_{0} \sqrt{1+v_{0}^{2} h_{0}^{2}} .
$$

Using (89), (90), (91), we obtain

$$
\begin{aligned}
\lim _{m \rightarrow 0} H_{2}(m) & =\pi+\frac{L}{2 n} \cdot\left(2 h_{0}\right)-\frac{L}{2 n} \cdot\left(2 h_{0} \sqrt{1+v_{0}^{2} h_{0}^{2}}\right) \\
& =\pi+\frac{L}{2 n} \cdot\left(\frac{2 n \pi}{L}\right)-\frac{L}{2 n} \cdot\left(\frac{2 n \pi}{L} \sqrt{1+v_{0}^{2} h_{0}^{2}}\right) \\
& =2 \pi-\pi \sqrt{1+v_{0}^{2} h_{0}^{2}} .
\end{aligned}
$$

The relation $\lim _{m \rightarrow 0} H_{2}(m)=\pi / n$ implies $v_{0}^{2} h_{0}^{2}=(3 n-1)(n-1) / n^{2}$. Substituting this to

$$
p=8 h_{0}^{5} v_{0}^{2} \sqrt{1+h_{0}^{2} v_{0}^{2}}
$$

we have

$$
p=\frac{8 \pi^{3}}{L^{3}}(n-1)(2 n-1)(3 n-1)
$$


Note that $Z_{1}(w):=2 \pi-\pi \sqrt{1+w}$ is monotone decreasing with respect to $w$ and $Z_{2}(w):=8 h^{3} w \sqrt{1+w}$ is monotone increasing. Therefore we obtain the assertion of the lemma.

The following proposition proves the second half of Theorem 2 .

Proposition 2. Let $n$ be an arbitrary integer with $n \geq 2$. Then a necessary and sufficient condition for the equations (12)-(14) to have a nontrivial solution of the form (19) (ii) is

$$
\frac{8 \pi^{3}}{L^{3}}(n-1)(2 n-1)(3 n-1)<p .
$$

If $n=1$, (12)-(14) does not have any nontrivial solution of the form (19) (ii).

Proof. Let $n \geq 2$.

(I) If $p<8 \pi^{3}(n-1)(2 n-1)(3 n-1) / L^{3}$, then, from the inequality $\lim _{m \rightarrow 0} H_{2}(m)>\pi / n$ (Lemma 33) and the fact that $H_{2}(m)$ is monotone increasing with respect to $m$ (Lemmas 30 and 32), (12) to (14) do not have nontrivial solutions of the form (19) (ii).

(II) If $p=8 \pi^{3}(n-1)(2 n-1)(3 n-1) / L^{3}$, then $H_{2}(m)=\pi / n$ has a unique solution $m=0$. However, if $m=0$, then $\kappa(s)=1 /(a \operatorname{sn}(K(0)-h(0) s)+b)+c=$ $1 /(a \cos (2 n \pi s / L)+b)+c$. Therefore this case is included in Case (i) of Lemma 7.

(III) If $p>8 \pi^{3}(n-1)(2 n-1)(3 n-1) / L^{3}$, then from the inequality $\lim _{m \rightarrow 0} H_{2}(m)<\pi / n$ and the monotonicity of $H_{2}(m)$, there uniquely exists $m^{*}$ $\left(0<m^{*}<1\right)$ such that $H_{2}\left(m^{*}\right)=\pi / n$. Since by (69), we already know that $a^{(-)}<0, b$, and $c$ are functions of $m$, so we have proven that there exists a unique nontrivial solution of the form (19) (ii).

If $n=1$, by Lemma 29 and the monotonicity of $H_{2}(m)$ it holds that $H_{2}(m)<\pi$ for arbitrary $m$ satisfying $0 \leq m<1$, and $p>0$. Therefore, in this case the equation $H_{2}(m)=\pi$ does not have any solution.

Combining Propositions 1 and 2, we have proven Theorem 2.

\section{Numerical experiment}

Fig. 10 is the $p$ versus $E(\Gamma)$ diagram drawn numerically according to the argument of Section 4. Each point of curves corresponds to a solution of (12) to (14). When $p=5.247$ and 21.65, which were computed by Flaherty, Keller and Rubinow [7], the solutions contacts with themselves. In addition, we see that $E(\Gamma)$ seems to have the same value when $p=\frac{8 \pi^{3}}{L^{3}}\left(n^{2}-1\right)$ and $p=\frac{8 \pi^{3}}{L^{3}}(n-1)(2 n-1)(3 n-1)$. Finally, we prove this fact.

Proposition 3. $E(\Gamma)$ takes the same value at $p=8 \pi^{3}\left(n^{2}-1\right) / L^{3}$ and $p=$ $8 \pi^{3}(n-1)(2 n-1)(3 n-1) / L^{3}$.

Proof. When $p=\frac{8 \pi^{3}}{L^{3}}\left(n^{2}-1\right)$, by Proposition 1 Case (2), the stationary closed curve (which satisfies $(12)-(14)$ ) is a disk of radius $L /(2 \pi)$. So, in this 


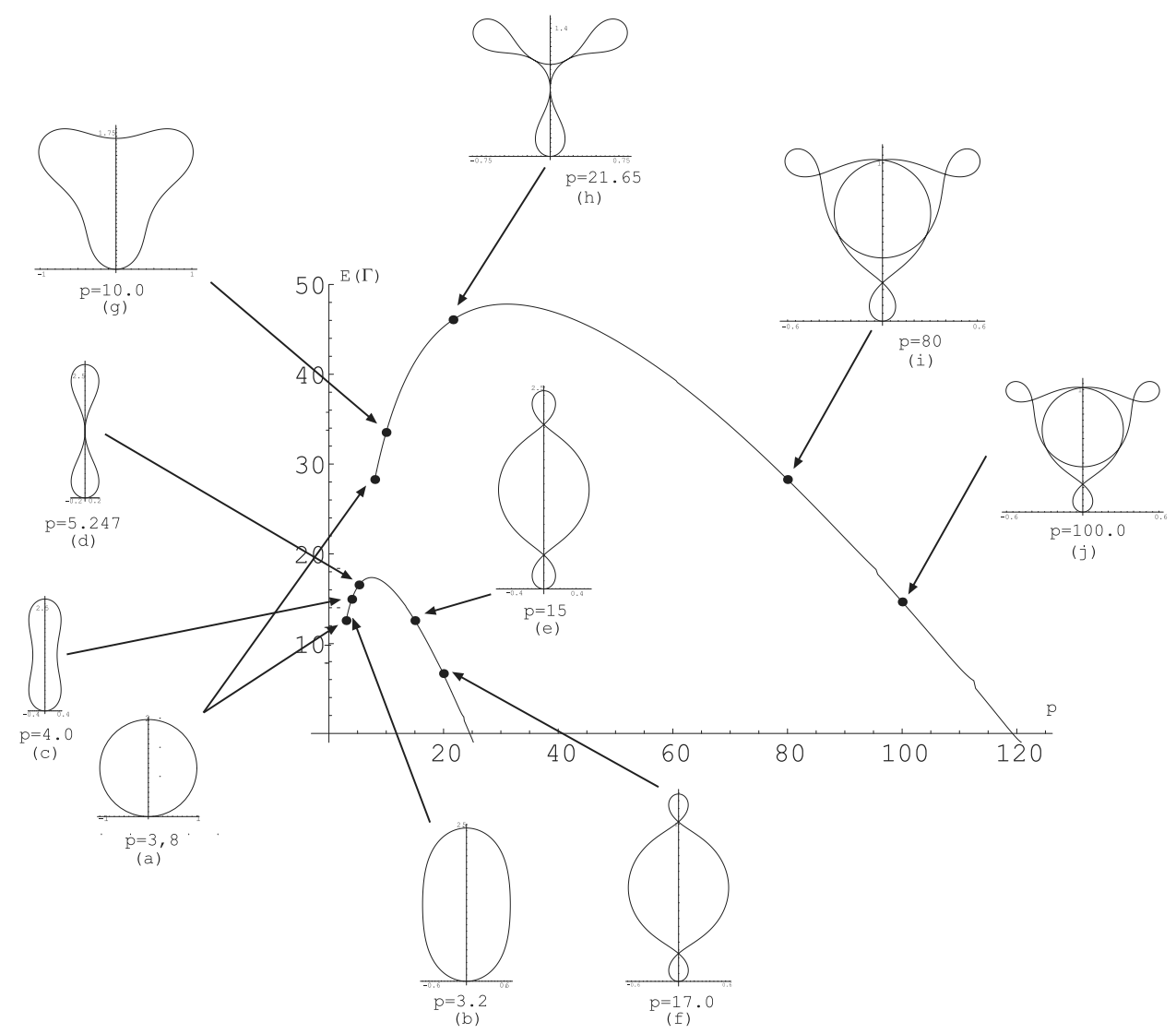

Fig. 10. The graph of $p$ versus $E(\Gamma)$ for $n=2$ and $n=3$ when $L=2 \pi$.

case $E(\Gamma)=2 \pi^{2} n^{2} / L$. By $[16$, p. 466$]$, the following equality holds:

$$
p M=\frac{1}{4} \int_{0}^{L} \kappa^{2}(s) d s-\frac{q_{1} L}{2} .
$$

Hence, $E(\Gamma)$ can be obtained without computing $\operatorname{Area}(\Omega)$ as

$$
E(\Gamma)=\frac{3}{4} \int_{0}^{L} \kappa^{2}(s) d s-\frac{q_{1} L}{2},
$$

where $q_{1}$ satisfies $(32)$. When $p=\frac{8 \pi^{3}}{L^{3}}(n-1)(2 n-1)(3 n-1)$, by Proposition 1 Case $(\mathrm{V})$ the curvature of the stationary closed curve takes the form

$$
\kappa(s)=\frac{1}{a \cos \left(\frac{2 n \pi s}{L}\right)+b}+c,
$$


where $a=-L \sqrt{(n-1)(3 n-1)} /\left(4 n^{2} \pi\right), b=L(2 n-1) /\left(4 n^{2} \pi\right), c=-2 \pi(2 n-1) / L$. Using the formula

$$
\int_{0}^{L}\left(\frac{1}{a \cos \left(\frac{2 n \pi s}{L}\right)+b}\right)^{2} d s=\frac{3 b L}{\left(b^{2}-a^{2}\right)^{\frac{3}{2}}}
$$

we obtain $E(\Gamma)=2 \pi^{2} n^{2} / L$.

\section{Appendix A. Proof of Lemma 5}

Let $\min _{0 \leq s \leq L / 2 n} \kappa(s)=\kappa(0)=\alpha$ and $\max _{0 \leq s \leq L / 2 n} \kappa(s)=\kappa(L / 2 n)=\beta$. Then (12) is expressed as

$$
\left(\frac{d \kappa}{d s}\right)^{2}=\frac{1}{4}(\beta-\kappa)(\kappa-\alpha)\left\{\kappa^{2}+(\alpha+\beta) \kappa+\gamma\right\},
$$

and $\left(p, q_{1}, q_{2}\right)$ is related to $(\alpha, \beta, \gamma)$ by

$$
q_{1}=\frac{1}{4}\left(\alpha^{2}+\beta^{2}+\alpha \beta-\gamma\right), \quad q_{2}=-\frac{1}{8} \alpha \beta \gamma, \quad p=\frac{1}{8}(\gamma-\alpha \beta)(\alpha+\beta) .
$$

Putting

$$
P_{1}(x)=x^{2}-(\alpha+\beta) x+\alpha \beta, \quad P_{2}(x)=x^{2}+(\alpha+\beta) x+\gamma,
$$

we have from (99)

$$
s=2 \int_{\alpha}^{\kappa} \frac{d x}{\sqrt{-P_{1}(x) P_{2}(x)}} .
$$

Since $P_{2}(x)=0$ should not have any root on the interval $[\alpha, \beta]$ (if $P_{2}(\alpha)=0$ or $P_{2}(\beta)=0$, the equation (99) has no periodic solution (see Arnold [3, Section 12])), it holds that

$$
\gamma+\alpha \beta+2 \alpha^{2}>0, \quad \gamma+\alpha \beta+2 \beta^{2}>0 .
$$

The right hand side of (100) will be reduced to the canonical form of real elliptic integrals according to the following three cases: (A) $\alpha+\beta>0$, (B) $\alpha+\beta<0$, and (C) $\alpha+\beta=0$. If (A) or (B), from (101), we see that the quadratic equation

$$
2(\alpha+\beta) t^{2}-2(\alpha \beta-\gamma) t-(\alpha+\beta)(\alpha \beta+\gamma)=0
$$

has distinct real roots $t_{0}$ and $t_{1}$, because the discriminant $D=\left(\gamma+\alpha \beta+2 \alpha^{2}\right)(\gamma+$ $\left.\alpha \beta+2 \beta^{2}\right)$ is positive. Assuming $t_{0}<t_{1}$, we have the following relation among $\alpha$, $\beta, t_{0}$ and $t_{1}$ :

$$
\begin{cases}t_{0}<\alpha<t_{1}<\beta & \text { in Case (A) } \\ \alpha<t_{0}<\beta<t_{1} & \text { in Case (B). }\end{cases}
$$


Let us prove only Case (A). In this case, we have

$$
t_{0}=\frac{\alpha \beta-\gamma-\sqrt{D}}{2(\alpha+\beta)}, \quad t_{1}=\frac{\alpha \beta-\gamma+\sqrt{D}}{2(\alpha+\beta)} .
$$

So, we obtain $t_{0}<\alpha$ if and only if $-\sqrt{D}<\gamma+\alpha \beta+2 \alpha^{2}, \alpha<t_{1}$ if and only if $\gamma+\alpha \beta+2 \alpha^{2}<\sqrt{D}$ which is equivalent to $\alpha+\beta>0$, and $t_{1}<\beta$ if and only if $\sqrt{D}<\gamma+\alpha \beta+2 \beta^{2}$, which is equivalent to $\alpha+\beta>0$. Keeping this relation in mind, we change the variable $x$ to $y$ as follows:

$$
\left\{\begin{array}{l}
x=\frac{t_{1}+t_{0} y}{1+y} \quad \text { in Case (A), } \\
x=\frac{t_{0}+t_{1} y}{1+y} \quad \text { in Case (B). }
\end{array}\right.
$$

Since $t_{0}+t_{1}=(\alpha \beta-\gamma) /(\alpha+\beta)$ and $t_{0} t_{1}=-(\alpha \beta+\gamma) / 2$, we have

$$
P_{i}(x)=\frac{P_{i}\left(t_{1}\right)+P_{i}\left(t_{0}\right) y^{2}}{(1+y)^{2}} \quad(i=1,2) .
$$

Let $y_{0} \in \mathbb{R}$ satisfy $\alpha=\left(t_{1}+t_{0} y_{0}\right) /\left(1+y_{0}\right)$. Then $\beta=\left(t_{1}-t_{0} y_{0}\right) /\left(1-y_{0}\right)$, because

$$
P_{1}\left(\frac{t_{1}-t_{0} y_{0}}{1-y_{0}}\right)=\frac{P_{1}\left(t_{1}\right)+P_{1}\left(t_{0}\right) y_{0}^{2}}{\left(1-y_{0}\right)^{2}}=\left(\frac{1+y_{0}}{1-y_{0}}\right)^{2} P_{1}(\alpha)=0
$$

and $y_{0} \neq 1$ by (101). Hence (104) (A) gives a bijection from the interval $[\alpha, \beta]$ to $\left[-y_{0}, y_{0}\right]$. For arbitrary $\kappa \in[\alpha, \beta]$, let $y_{1} \in \mathbb{R}$ be the element satisfying $\kappa=$ $\left(t_{1}+t_{0} y_{1}\right) /\left(1+y_{1}\right)$. Then we have from $(100)$

$$
s=2 \int_{y_{0}}^{y_{1}} \frac{\left(t_{0}-t_{1}\right) d y}{\sqrt{-\left(P_{1}\left(t_{1}\right)+P_{1}\left(t_{0}\right) y^{2}\right)\left(P_{2}\left(t_{1}\right)+P_{2}\left(t_{0}\right) y^{2}\right)}} .
$$

This equation will be further made simpler according to the following three cases:

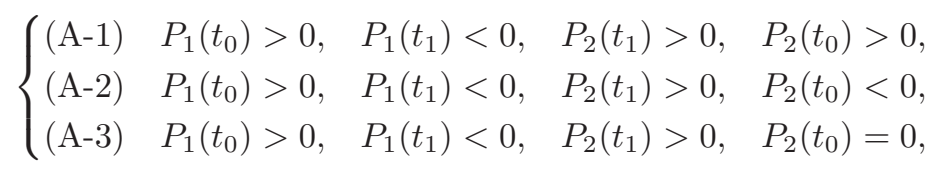

since $t_{0}<\alpha<t_{1}<\beta$, it holds that $P_{1}\left(t_{0}\right)>0, P_{1}\left(t_{1}\right)<0$, and $P_{2}(x)>0$ on $[\alpha, \beta]$.

When (A-1) holds, we have from (106)

$$
s=\frac{2\left(t_{0}-t_{1}\right)}{\sqrt{P_{1}\left(t_{0}\right) P_{2}\left(t_{0}\right)}} \int_{y_{0}}^{y_{1}} \frac{d y}{\sqrt{\left(-\frac{P_{1}\left(t_{1}\right)}{P_{1}\left(t_{0}\right)}-y^{2}\right)\left(\frac{P_{2}\left(t_{1}\right)}{P_{2}\left(t_{0}\right)}+y^{2}\right)}} .
$$


If $0 \leq y_{1} \leq y_{0}$, then putting $y=\sqrt{-\left(1-z^{2}\right) P_{1}\left(t_{1}\right) / P_{1}\left(t_{0}\right)}$, we have

$$
s=\frac{1}{h} \int_{0}^{z_{1}} \frac{d z}{\sqrt{\left(1-z^{2}\right)\left(1-m z^{2}\right)}},
$$

where

$$
\begin{aligned}
m & =\frac{-P_{1}\left(t_{1}\right) P_{2}\left(t_{0}\right)}{P_{1}\left(t_{0}\right) P_{2}\left(t_{1}\right)-P_{1}\left(t_{1}\right) P_{2}\left(t_{0}\right)}, \quad h=\frac{\sqrt{P_{1}\left(t_{0}\right) P_{2}\left(t_{1}\right)-P_{1}\left(t_{1}\right) P_{2}\left(t_{0}\right)}}{2\left(t_{1}-t_{0}\right)}, \\
y_{1} & =\sqrt{-\frac{P_{1}\left(t_{1}\right)}{P_{1}\left(t_{0}\right)}\left(1-z_{1}^{2}\right)} .
\end{aligned}
$$

From (108), the range of $s$ becomes $[0, K(m) / h]$ and $z_{1}$ is represented as $z_{1}=$ $\operatorname{sn}(h s)$. Therefore,

$$
y_{1}=\sqrt{-\frac{P_{1}\left(t_{1}\right)}{P_{1}\left(t_{0}\right)}} \mathrm{cn}(h s),
$$

and hence

$$
\kappa(s)=\frac{t_{1}+t_{0} \sqrt{-\frac{P_{1}\left(t_{1}\right)}{P_{1}\left(t_{0}\right)}} \operatorname{cn}(h s)}{1+\sqrt{-\frac{P_{1}\left(t_{1}\right)}{P_{1}\left(t_{0}\right)}} \operatorname{cn}(h s)} .
$$

If $-y_{0} \leq y_{1} \leq 0$, then

$$
\begin{aligned}
s= & \frac{2\left(t_{0}-t_{1}\right)}{\sqrt{P_{1}\left(t_{0}\right) P_{2}\left(t_{0}\right)}} \\
& \times\left(\int_{y_{0}}^{0} \frac{d y}{\sqrt{\left(-\frac{P_{1}\left(t_{1}\right)}{P_{1}\left(t_{0}\right)}-y^{2}\right)\left(\frac{P_{2}\left(t_{1}\right)}{P_{2}\left(t_{0}\right)}+y^{2}\right)}}+\int_{0}^{y_{1}} \frac{d y}{\sqrt{\left(-\frac{P_{1}\left(t_{1}\right)}{P_{1}\left(t_{0}\right)}-y^{2}\right)\left(\frac{P_{2}\left(t_{1}\right)}{P_{2}\left(t_{0}\right)}+y^{2}\right)}}\right) .
\end{aligned}
$$

Putting $y=-\sqrt{-\left(1-z^{2}\right) P_{1}\left(t_{1}\right) / P_{1}\left(t_{0}\right)}$, we have

$$
\begin{aligned}
s & =\frac{K(m)}{h}-\frac{1}{h} \int_{1}^{z_{1}} \frac{d z}{\sqrt{\left(1-z^{2}\right)\left(1-m z^{2}\right)}} \\
& =\frac{2 K(m)}{h}-\frac{1}{h} \int_{0}^{z_{1}} \frac{d z}{\sqrt{\left(1-z^{2}\right)\left(1-m z^{2}\right)}}
\end{aligned}
$$

where $m$ and $h$ are the same as in the case $0 \leq y_{1} \leq y_{0}$ and

$$
y_{1}=-\sqrt{-\frac{P_{1}\left(t_{1}\right)}{P_{1}\left(t_{0}\right)}\left(1-z_{1}^{2}\right)} .
$$

Therefore the range of $s$ becomes $[K(m) / h, 2 K(m) / h]$ and $z_{1}$ is represented as $z_{1}=\operatorname{sn}(2 K(m)-h s)=\operatorname{sn}(h s)$. Noting that $\operatorname{cn}(h s)<0$ on $[K(m) / h, 2 K(m) / h]$, we see that $y_{1}$ and $\kappa$ is again represented as (109) and (110) respectively. 
When (A-2) holds, we have from (106)

$$
s=\frac{2\left(t_{0}-t_{1}\right)}{\sqrt{-P_{1}\left(t_{0}\right) P_{2}\left(t_{0}\right)}} \int_{y_{0}}^{y_{1}} \frac{d y}{\sqrt{\left(-\frac{P_{1}\left(t_{1}\right)}{P_{1}\left(t_{0}\right)}-y^{2}\right)\left(-\frac{P_{2}\left(t_{1}\right)}{P_{2}\left(t_{0}\right)}-y^{2}\right)}} .
$$

If $0 \leq y_{1} \leq y_{0}$, then putting $y=\sqrt{-P_{1}\left(t_{1}\right) / P_{1}\left(t_{0}\right)} z$, we have

$$
s=-\frac{1}{h} \int_{1}^{z_{1}} \frac{d z}{\sqrt{\left(1-z^{2}\right)\left(1-m z^{2}\right)}},
$$

where

$$
m=\frac{P_{1}\left(t_{1}\right) P_{2}\left(t_{0}\right)}{P_{1}\left(t_{0}\right) P_{2}\left(t_{1}\right)}, \quad h=\frac{\sqrt{P_{1}\left(t_{0}\right) P_{2}\left(t_{1}\right)}}{2\left(t_{1}-t_{0}\right)}, \quad y_{1}=\sqrt{-\frac{P_{1}\left(t_{1}\right)}{P_{1}\left(t_{0}\right)}} z_{1} .
$$

From (112), the range of $s$ becomes $[0, K(m) / h]$ and $z_{1}$ is represented as $z_{1}=$ $\operatorname{sn}(K(m)-h s)$. Therefore,

$$
y_{1}=\sqrt{-\frac{P_{1}\left(t_{1}\right)}{P_{1}\left(t_{0}\right)}} \operatorname{sn}(K(m)-h s)
$$

and hence

$$
\kappa(s)=\frac{t_{1}+t_{0} \sqrt{-\frac{P_{1}\left(t_{1}\right)}{P_{1}\left(t_{0}\right)}} \operatorname{sn}(K(m)-h s)}{1+\sqrt{-\frac{P_{1}\left(t_{1}\right)}{P_{1}\left(t_{0}\right)}} \operatorname{sn}(K(m)-h s)} .
$$

If $-y_{0} \leq y_{1} \leq 0$, then as in Case (A-1), dividing the interval of integration into $\left[y_{0}, 0\right]$ and $\left[0, y_{1}\right]$, and putting $y=-\sqrt{-P_{1}\left(t_{1}\right) / P_{1}\left(t_{0}\right)} z$, we see that (114) also holds on the interval $[K(m) / h, 2 K(m) / h]$.

When (A-3) holds, we have from (106)

$$
s=\frac{2\left(t_{0}-t_{1}\right)}{\sqrt{P_{1}\left(t_{0}\right) P_{2}\left(t_{1}\right)}} \int_{y_{0}}^{y_{1}} \frac{d y}{\sqrt{-\frac{P_{1}\left(t_{1}\right)}{P_{1}\left(t_{0}\right)}-y^{2}}}=\frac{1}{h} \arccos \left(\sqrt{-\frac{P_{1}\left(t_{0}\right)}{P_{1}\left(t_{1}\right)}} y_{1}\right),
$$

where $h=\sqrt{P_{1}\left(t_{0}\right) P_{2}\left(t_{1}\right)} /\left(2\left(t_{1}-t_{0}\right)\right)$. Thus

$$
y_{1}=\sqrt{-\frac{P_{1}\left(t_{1}\right)}{P_{1}\left(t_{0}\right)}} \cos (h s),
$$

and hence

$$
\kappa(s)=\frac{t_{1}+t_{0} \sqrt{-\frac{P_{1}\left(t_{1}\right)}{P_{1}\left(t_{0}\right)}} \cos (h s)}{1+\sqrt{-\frac{P_{1}\left(t_{1}\right)}{P_{1}\left(t_{0}\right)}} \cos (h s)} \quad\left(0 \leq s \leq \frac{\pi}{h}\right) .
$$

This is a special case $(m=0)$ of Case $(\mathrm{A}-1)$. Hence, it is not necessary to take Case (A-3) into account. 
Now we consider Case (B). Let $y_{0} \in \mathbb{R}$ satisfy $\beta=\left(t_{0}+t_{1} y_{0}\right) /\left(1+y_{0}\right)$. Then, as in Case (A), it holds that $\alpha=\left(t_{0}-t_{1} y_{0}\right) /\left(1-y_{0}\right)$. Let $y_{1} \in \mathbb{R}$ be the element satisfying $\kappa=\left(t_{0}+t_{1} y_{1}\right) /\left(1+y_{1}\right)$, then we have from (100)

$$
s=2 \int_{-y_{0}}^{y_{1}} \frac{\left(t_{1}-t_{0}\right) d y}{\sqrt{-\left(P_{1}\left(t_{0}\right)+P_{1}\left(t_{1}\right) y^{2}\right)\left(P_{2}\left(t_{0}\right)+P_{2}\left(t_{1}\right) y^{2}\right)}} .
$$

This equation will be further simplified according to the following three cases

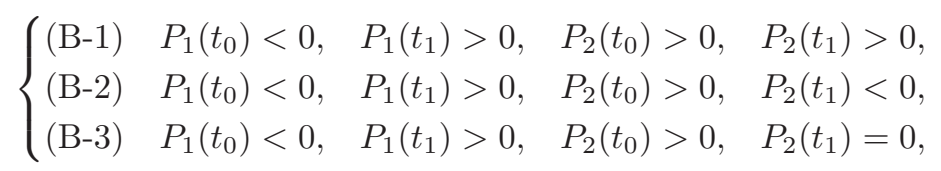

since $\alpha<t_{0}<\beta<t_{1}, P_{1}\left(t_{0}\right)<0, P_{1}\left(t_{1}\right)>0$, and $P_{2}(x)>0$ on $[\alpha, \beta]$. Applying the same argument as Case (A),

$$
\begin{aligned}
& \kappa(s)=\frac{t_{0}-t_{1} \sqrt{-\frac{P_{1}\left(t_{0}\right)}{P_{1}\left(t_{1}\right)}} \mathrm{cn}(h s)}{1-\sqrt{-\frac{P_{1}\left(t_{0}\right)}{P_{1}\left(t_{1}\right)}} \mathrm{cn}(h s)}, \quad \text { where }\left\{\begin{array}{l}
m=\frac{-P_{2}\left(t_{1}\right) P_{1}\left(t_{0}\right)}{P_{2}\left(t_{0}\right) P_{1}\left(t_{1}\right)-P_{1}\left(t_{0}\right) P_{2}\left(t_{1}\right)}, \\
h=\frac{\sqrt{P_{2}\left(t_{0}\right) P_{1}\left(t_{1}\right)-P_{1}\left(t_{0}\right) P_{2}\left(t_{1}\right)}}{2\left(t_{1}-t_{0}\right)},
\end{array}\right. \\
& \kappa(s)=\frac{t_{0}-t_{1} \sqrt{-\frac{P_{1}\left(t_{0}\right)}{P_{1}\left(t_{1}\right)}} \operatorname{sn}(K(m)-h s)}{1-\sqrt{-\frac{P_{1}\left(t_{0}\right)}{P_{1}\left(t_{1}\right)}} \operatorname{sn}(K(m)-h s)}, \quad \text { where }\left\{\begin{array}{l}
m=\frac{P_{1}\left(t_{0}\right) P_{2}\left(t_{1}\right)}{P_{2}\left(t_{0}\right) P_{1}\left(t_{1}\right)}, \\
h=\frac{\sqrt{P_{1}\left(t_{1}\right) P_{2}\left(t_{0}\right)}}{2\left(t_{1}-t_{0}\right)},
\end{array}\right. \\
& \kappa(s)=\frac{t_{0}-t_{1} \sqrt{-\frac{P_{1}\left(t_{0}\right)}{P_{1}\left(t_{1}\right)}} \cos (h s)}{1-\sqrt{-\frac{P_{1}\left(t_{0}\right)}{P_{1}\left(t_{1}\right)}} \cos (h s)}, \quad \text { where } h=\frac{\sqrt{P_{1}\left(t_{1}\right) P_{2}\left(t_{0}\right)}}{2\left(t_{1}-t_{0}\right)}
\end{aligned}
$$

are obtained.

Finally, we consider Case (C). In this case, from (100) it holds that

$$
s=2 \int_{-\beta}^{\kappa} \frac{1}{\sqrt{\left(\beta^{2}-x^{2}\right)\left(x^{2}+\gamma\right)}},
$$

where $\gamma>0$ (since $x^{2}+\gamma$ does not have any root on $\left.[-\beta, \beta]\right)$.

If $-\beta \leq \kappa \leq 0$, then putting $x=-\beta \sqrt{1-z^{2}}$, we have

$$
s=\frac{2}{\sqrt{\beta^{2}+\gamma}} \int_{0}^{z_{1}} \frac{1}{\sqrt{\left(1-z^{2}\right)\left(1-m z^{2}\right)}},
$$

where $m=\beta^{2} /\left(\beta^{2}+\gamma\right)$ and $\kappa=-\beta \sqrt{1-z_{1}^{2}}$. From (119), the range of $s$ is $0 \leq s \leq 2 K(m) / \sqrt{\beta^{2}+\gamma}$, and $z_{1}=\operatorname{sn}\left(\sqrt{\beta^{2}+\gamma} s / 2\right)$. Thus,

$$
\kappa(s)=-\beta \operatorname{cn}\left(\frac{\sqrt{\beta^{2}+\gamma}}{2} s\right) .
$$


If $0 \leq \kappa \leq \beta$, then putting $x=\beta \sqrt{1-z^{2}}$, we have (120) again (in this case, the range of $s$ becomes $\left.2 K(m) / \sqrt{\beta^{2}+\gamma} \leq s \leq 4 K(m) / \sqrt{\beta^{2}+\gamma}\right)$.

Now we have completed the proof of Lemma 5 .

\section{Appendix B. Proof of Lemma 6}

Let us choose $\gamma=\alpha \beta$ in (99) and assume $0<\alpha<\beta$. Then (100) becomes

$$
s=2 \int_{\alpha}^{\kappa} \frac{d x}{\sqrt{\left(x^{2}-\alpha^{2}\right)\left(\beta^{2}-x^{2}\right)}} .
$$

In this case, (102) has two roots $t_{0}=-\sqrt{\alpha \beta}$ and $t_{1}=\sqrt{\alpha \beta}$. Since $P_{2}\left(t_{0}\right)=$ $-\sqrt{\alpha \beta}(\sqrt{\beta}-\sqrt{\alpha})^{2}<0$, this case applies to Case (A-2), so, we have from (114)

$$
\kappa(s)=\frac{\sqrt{\alpha \beta}-\sqrt{\alpha \beta}\left(\frac{\sqrt{\beta}-\sqrt{\alpha}}{\sqrt{\beta}+\sqrt{\alpha}}\right) \operatorname{sn}(K(m)-h s, m)}{1+\left(\frac{\sqrt{\beta}-\sqrt{\alpha}}{\sqrt{\beta}+\sqrt{\alpha}}\right) \operatorname{sn}(K(m)-h s, m)},
$$

where

$$
m=\left(\frac{\sqrt{\beta}-\sqrt{\alpha}}{\sqrt{\beta}+\sqrt{\alpha}}\right)^{4}, \quad h=\left(\frac{\sqrt{\beta}+\sqrt{\alpha}}{2}\right)^{2} .
$$

On the other hand, putting $x^{2}=\beta^{2}-\left(\beta^{2}-\alpha^{2}\right) z^{2}$, we see that (121) becomes

$$
s=-2 \int_{1}^{z_{1}} \frac{d z}{\beta \sqrt{\left(1-z^{2}\right)\left(1-\frac{\beta^{2}-\alpha^{2}}{\beta^{2}} z^{2}\right)}},
$$

where $z_{1}$ satisfies $\kappa^{2}=\beta^{2}-\left(\beta^{2}-\alpha^{2}\right) z_{1}^{2}$. Therefore,

$$
z_{1}=\operatorname{sn}\left(K\left(m_{0}\right)-\frac{\beta s}{2}, m_{0}\right)=\frac{\operatorname{cn}\left(\frac{\beta s}{2}, m_{0}\right)}{\operatorname{dn}\left(\frac{\beta s}{2}, m_{0}\right)},
$$

where $m_{0}=\frac{\beta^{2}-\alpha^{2}}{\beta^{2}}$. Hence

$$
\begin{aligned}
\kappa^{2}(s) & =\frac{\beta^{2}\left(1-\frac{\beta^{2}-\alpha^{2}}{\beta^{2}} \operatorname{sn}^{2}\left(\frac{\beta s}{2}, m_{0}\right)\right)-\left(\beta^{2}-\alpha^{2}\right) \operatorname{cn}^{2}\left(\frac{\beta s}{2}, m_{0}\right)}{\operatorname{dn}^{2}\left(\frac{\beta s}{2}, m_{0}\right)} \\
& =\frac{\alpha^{2}}{\operatorname{dn}^{2}\left(\frac{\beta s}{2}, m_{0}\right)} .
\end{aligned}
$$

Thus we have

$$
\kappa(s)=\frac{\alpha}{\operatorname{dn}\left(\frac{\beta s}{2}, m_{0}\right)} .
$$


Comparing (122) with (124), we have

$$
\operatorname{sn}(K(m)-h s, m)=\frac{\sqrt{\beta}+\sqrt{\alpha}}{\sqrt{\beta}-\sqrt{\alpha}} \cdot \frac{\sqrt{\beta} \operatorname{dn}\left(\frac{\beta s}{2}, m_{0}\right)-\sqrt{\alpha}}{\sqrt{\beta} \operatorname{dn}\left(\frac{\beta s}{2}, m_{0}\right)+\sqrt{\alpha}} .
$$

From (123), we have $\alpha=h\left(1-m^{\frac{1}{4}}\right)^{2}, \beta=h\left(1+m^{\frac{1}{4}}\right)^{2}$. Substituting these to (125), we obtain the result.

Acknowledgement. The authors would like to express hearty gratitude to an anonymous referee who gave them many valuable suggestions. This work was supported in part by the Grant-in-Aid for Scientific Research (A) 18204010, Japan Society for the Promotion of Science.

\section{References}

[1] M. Abramowitz and I.A. Stegun, Handbook of mathematical functions with formulas, graphs, and mathematical tables. Dover, New York, 1972.

[2] S.S. Antman, Nonlinear Problems of Elasticity. Applied Mathematical Sciences, 107, Springer, New York, 1995.

[ 3 ] V.I. Arnold, Ordinary Differential Equations. The M.I.T. Press, Cambridge, 1973.

[ 4 ] G. Arreaga, R. Capovilla, C. Chryssomalakos and J. Guven, Area-constrained planar elastica. Physical Review E, 65 (2002), 031801.

[5] P. Canham, The minimum energy of bending as a possible explanation of the biconcave shape of the human red blood cell. J. Theor. Biol., 26 (1970), 61-81.

[ 6 ] G.F. Carrier, On the buckling of elastic rings. J. Math. Phys. Mass. Inst. Tech., 26 (1947), 94-103.

[ 7 ] J.E. Flaherty, J.B. Keller and S.I. Rubinow, Post buckling behavior of elastic tubes and rings with opposite side in contact. SIAM J. Appl. Math., 23 (1972), 446-455.

[ 8 ] M. Gage, An isoperimetric inequality with applications to curve shortening. Duke Math. J., 50 (1983), 1225-1229.

[9 ] C.G.J. Jacobi, Theorie der Elliptischen Functionen aus den Eigenshaften der Thetareihen abgeleitet. Gesammelte Werke, Vol. 1, 495-538.

[10] M. Kac, Can one hear the shape of a drum? Amer. Math. Monthly, 73 (1966), 1-23.

[11] W. Matsumoto, M. Murai and S. Yotsutani, By which kind of sound, can one hear the shape of a drum? RIMS Kokyuroku, 1315 (2003), 156-175.

[12] S. Okabe, Asymptotic form of solutions of the Tadjbakhsh-Odeh variational problem. Advanced Studies in Pure Mathematics, 47 (2007), 709-728.

[13] K. Satake and H. Honda, Evolution towards the solution of a shape optimization problem. Forma, 17 (2002), 253-274.

[14] I. Tadjbakhsh and F. Odeh, Equilibrium states of elastic rings. J. Math. Anal. Appl., 18 (1967), 59-74.

[15] C. Truesdell, The influence of elasticity on analysis: the classic heritage. Bull. Amer. Math. Soc. (N.S.), 9 (1983), 293-310.

[16] K. Watanabe, Plane domains which are spectrally determined. Ann. Grobal Anal. Geom., 18 (2000), 447-475.

[17] E.T. Whittaker and G.N. Watson, A Course of Modern Analysis. Cambridge University Press, Cambridge, 1935.

[18] http://functions.wolfram.com/EllipticIntegrals/EllipticPi/20/01/ShowAll.html. 